\title{
A General Asymmetric Synthesis of (R)-Matsutakeol and Flavored Analogs
}

\author{
Jia Liu ${ }^{1}$, Honglian Li ${ }^{1}$, Chao Zheng ${ }^{2}$, Shichao Lu ${ }^{1}$, Xianru Guo ${ }^{1}$, Xinming Yin ${ }^{1}$, Risong Na ${ }^{1,4, *}$, \\ Bin $\mathrm{Yu}^{3, *}$ and Min Wang ${ }^{4}$ \\ 1 Collaborative Innovation Center of Henan Grain Crops, National Key Laboratory of Wheat and Maize Crop \\ Science, College of Plant Protection, Henan Agricultural University, Wenhua Road No. 95, \\ Zhengzhou 450002, China; ljia198346@163.com (J.L.); honglianli@sina.com (H.L.); \\ 15517151076@163.com (S.L.); guoxianru@126.com (X.G.); xinmingyin@hotmail.com (X.Y.) \\ 2 Key Laboratory of Tropical Medicinal Plant Chemistry of Hainan Province, School of Chemistry and \\ Chemical Engineering, Hainan Normal University, Haikou 57115, China; zhengchaoustc@163.com \\ 3 School of Pharmaceutical Sciences, Zhengzhou University, Zhengzhou 450001, China \\ 4 School of Sciences, China Agricultural University, Beijing 100193, China; wangmincau@outlook.com \\ * Correspondence: nrs@henau.edu.cn or risongna@163.com (R.N.); zzuyubin@hotmail.com (B.Y.); \\ Tel.: +86-371-6355-8170 (R.N.)
}

Academic Editor: Rafael Chinchilla

Received: 18 January 2017; Accepted: 24 February 2017; Published: 27 February 2017

\begin{abstract}
An efficient and practical synthetic route toward chiral matsutakeol and analogs was developed by asymmetric addition of terminal alkyne to aldehydes. (R)-matsutakeol and other flavored substances were feasibly synthesized from various alkylaldehydes in high yield (up to $49.5 \%$, in three steps) and excellent enantiomeric excess (up to $>99 \%$ ). The protocols may serve as an alternative asymmetric synthetic method for active small-molecule library of natural fatty acid metabolites and analogs. These chiral allyl alcohols are prepared for food analysis and screening insect attractants.
\end{abstract}

Keywords: $(R)$-matsutakeol; general method; asymmetric catalysis; mushrooms; flavored analogs

\section{Introduction}

Many natural unsaturated alcohols 1-6 (Figure 1) are very important fatty acid metabolites derived from fungi [1] and plants [2]. These substances have been found in nutrient, pharmaceutical, and agricultural use, as their structural diversity and variety of biological activities. For examples, the chiral allyl alcohols $\mathbf{6 a - c}$ (Figure 1) are a class of important flavor substances and dietary supplements which are widely used in the food industry [3-5]. (R)-matsutakeol 6a isolated from matsutake [6], has been found to possess antitumor properties [3]. Effects of inhibition on fungal spore germination and mushroom and plant development have also been discovered [7]. Recently, $(R)$-matsutakeol 6a and its analogs are widely used in insecticidal compositions as effective attractants for some harmful hematophagous insects $[8,9]$. In particular, the enantioselectivity and chiral configuration of the compounds directly determined biological activities such as smell and taste [10].

Inspired by potential applications above, great attention has been paid to preparation of compounds 6 with high enantioselectivity and structural diversity [11-19]. To take (R)-matsutakeol 6a as an example, in 1987, 6a and its enantiomer were obtained by Helmchen through the asymmetric retro Diels-Alder reaction [11]. In the same year, Takano prepared the $(R)$-matsutakeol by using the optically active starting material of $(R)$-epichlorohydrin [12]. In 1988, Kitamura obtained $(R)$-matsutakeol $(21 \% e e)$ via kinetic resolution of racemic allylic alcohols [13]. Oppolzer developed an enantioseletive synthesis of $(R)$-matsutakeol $(96 \%$ ee) by catalytic asymmetric addition of 
divinylzinc to hexanal [14]. In 2005, a chiral resolution of racemic matsutakeol was conducted using (S)-(+)-2-methoxy-2-(1-naphthyl) propionic acid (M $\alpha \mathrm{NP}$ acid) by Kusuda [15]. In addition, various enzymatic kinetic resolution and enzyme-catalyzed reactions have also been used in preparation of chiral matsutakeol. In 2010, Bisogno developed an efficient oxidoreductase-catalyzed system to obtain (R)-matsutakeol in $86 \%$ ee [16]. Recently, Rej prepared (S)-matsutakeol as an important intermediate, by ME-DKR (metal enzyme combined dynamic kinetic resolution) of $( \pm)$-oct-1-en-3-ol [17,18]. In 2016, Lee developed a synthetic strategy towards (S)-matsutakeol in 10 steps from inexpensive, natural (2S,3S)-D-tartaric acid with 32\% yield [19].

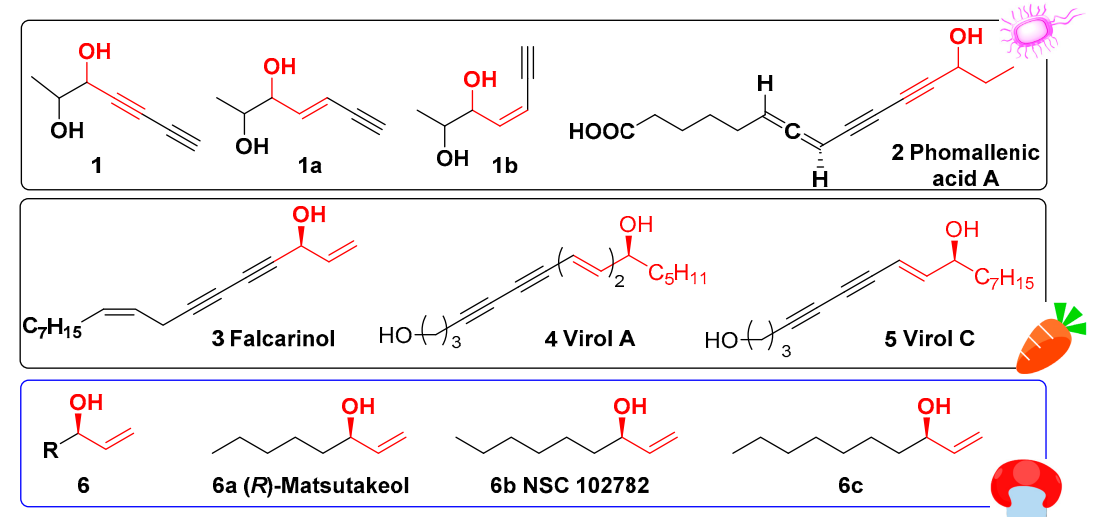

Figure 1. Natural unsaturated alcohols derived from fungi and plant.

In summary, matsutakeol could be prepared by chiral pool synthesis [19], enzymatic kinetic resolution [17,18], and asymmetric synthesis [14] (Scheme 1). Although, several synthetic routes have been developed, there is still a lack of efficient method for obtaining a highly enantioselective allyl alcohols library in screening for flavors or attractants. Compared to asymmetric synthesis (Scheme 1, method 3), the structural diversity of the reaction products is limited by chiral sources (Scheme 1, method 1) [19], and the effect of enzymatic reaction (Scheme 1, method 2) is constrained by stability and activity of enzymes $[17,18]$. By retro-synthetic analysis, the chiral olefinic alcohol 6 could be formed from propargyl alcohol 12, and chiral propargyl alcohol 12 can be synthesized from the corresponding methyl propiolate 13 (Scheme 1, method 3). Highly enantioselective methyl propiolate (S)-13 is obtained by Zn-catalyzed asymmetric addition of terminal alkynes $\mathbf{1 5}$ to various aldehydes 14. This reaction has been widely studied (Scheme 2) [20-50], in which numerous chiral ligands are applied, such as $(R, R)$-ProPhenol 16a [24,39], $(R)$-BINOL 17a, and its derivatives [28,30], $\beta$-Sulfonamide Alcohol 18 [31,32], Wolf's ligand 19 [41], and Wang's ligand 20 (our previous group research) [33]. Among the numerous catalysts, $(R, R)$-ProPhenol 16a and $(R)$-BINOL 17a are readily available and well-established [20-50].

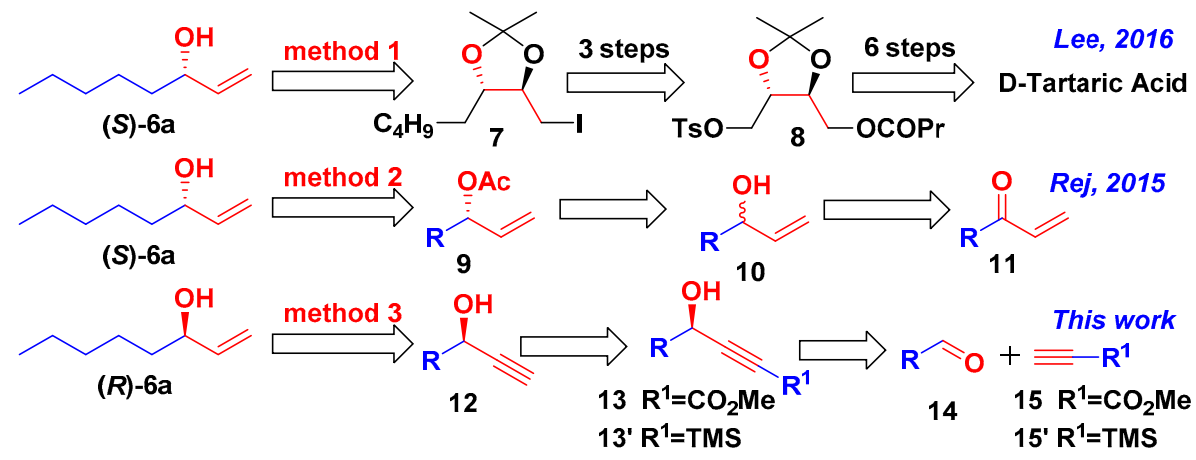

Scheme 1. Retro-synthetic analysis of matsutakeol and its natural analogs reported in the latest literature. 


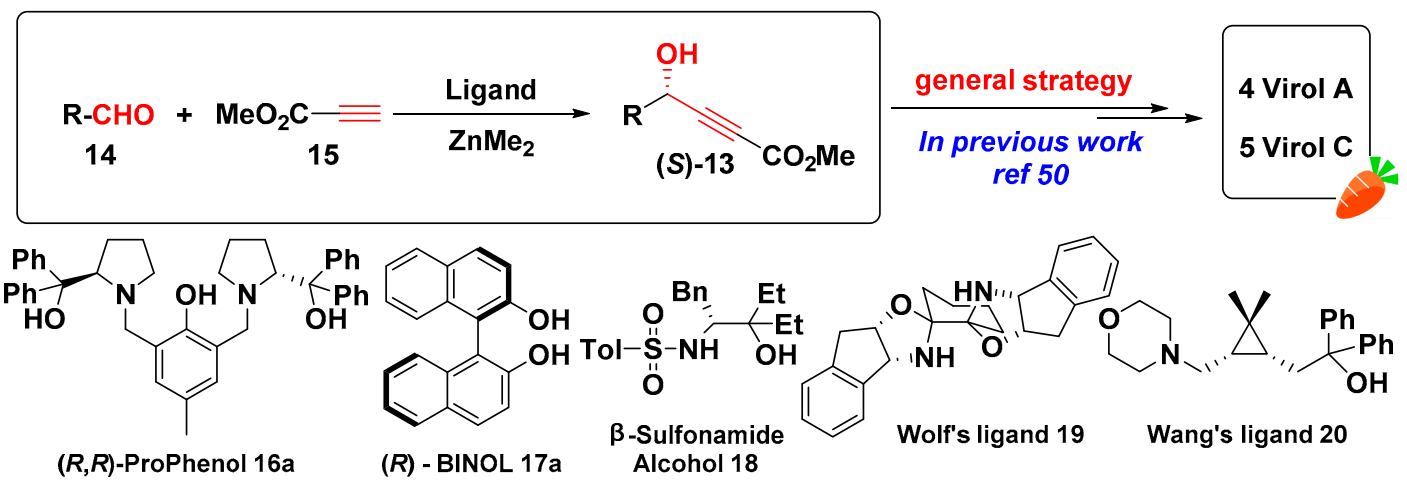

Scheme 2. Zn-catalyzed asymmetric direct addition of terminal alkynes to aldehydes.

\section{Results and Discussion}

Herein we report the asymmetric synthesis of $(R)$-matsutakeol and its natural analogs. In our previous work (Scheme 2) [50], the reaction conditions were optimized for the stereoselective addition of methyl propiolate to aliphatic aldehydes, and the highest enantiomeric excess values $(97 \%-99 \%$ ee) of $(S)$-alkynol product 13 were afforded by $(R, R)$-ProPhenol/Zn complex. It is developed in a general strategy toward the total synthesis of C17 polyacetylenes such as virol A and virol C [50].

On the basis of previous work $[24,25,39,50]$, we expanded substrate scope of the $(S, S)$-ProPhenolcatalyzed direct asymmetric addition, affording the corresponding $(R)$-configured propargyl alcohols 13 in moderated yields $(63 \%-73 \%)$ and with excellent enantioselectivities $(97 \%-99 \%$ ee) (Table 1$)$. Thus, we developed a general method with two steps to obtain highly enantioselective allyl alcohol flavors.

Table 1. Synthesis of chiral alkynol units 13a-e of C17 polyacetylenes via the asymmetric addition of methyl propiolate to aliphatic aldehydes ${ }^{a}$.
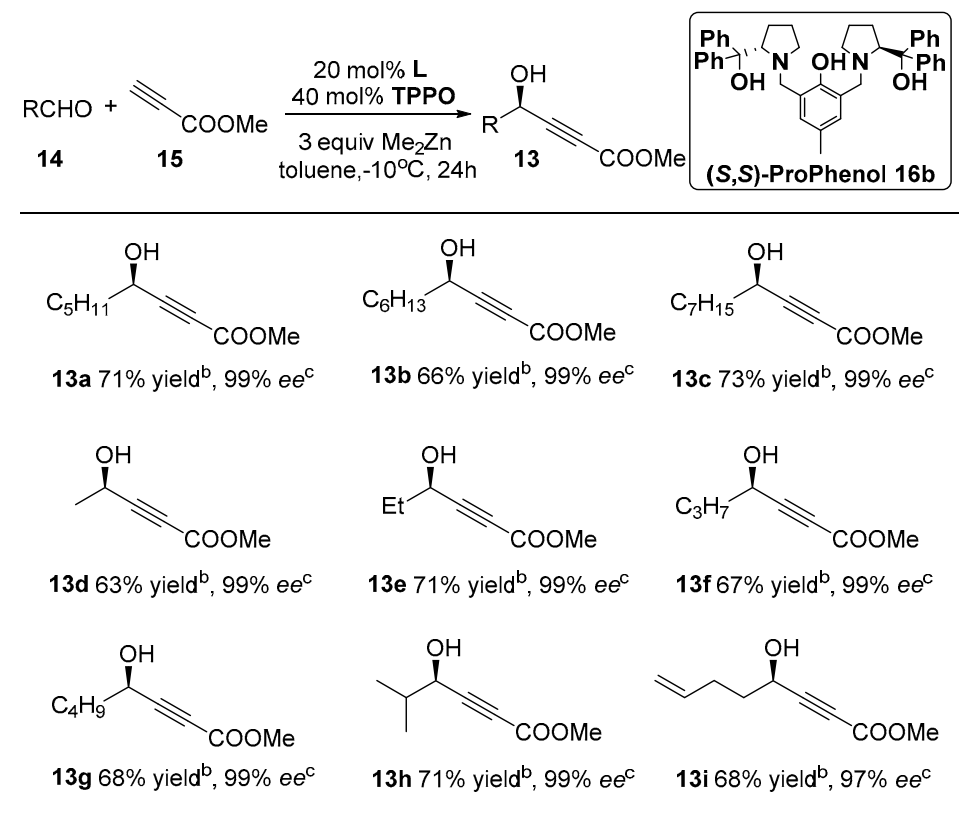

\footnotetext{
${ }^{\text {a }}$ Unless otherwise noted, the reaction was carried out on a $1.0 \mathrm{mmol}$ scale in toluene $(1.0 \mathrm{~mL}){ }^{\mathrm{b}}$ Isolated yields;

${ }^{c}$ The ee values were determined by chiral HPLC.
}

As shown in Scheme 3, treatment of compounds 13a-c with LiOH in THF gave the corresponding carboxylic acid intermediates [38], which were then subjected to the $\mathrm{CuCl}$-catalyzed decarboxylation directly, producing the chiral propargyl alcohols 12a-c in good yields (83\%-86\%) and without loss of 
enantio-selectivity (99\% ee). Reduction of compounds $\mathbf{1 2 a}-\mathbf{c}$ with $\mathrm{NaBH}_{4}$ in the presence of $\mathrm{Ni}(\mathrm{OAc})_{2}$ gave $(R)$-matsutakeol $\mathbf{6 a}$ and its natural analogs $\mathbf{6 b}$ and $\mathbf{6 c}$ in good yields (82\%-85\%) [51]. However, the ee values of $(R)$-matsutakeol and analogs could not be directly resolved by HPLC on a chiral column conveniently. Therefore, derivatization of compounds $\mathbf{6 a - c}$ through introducing 3,5-dinitrobenzoyl moiety to the natural molecules, directly afforded compounds $21 \mathbf{a}-\mathbf{c}$ in nearly quantitative yields $(93 \%-95 \%)$ and $99 \%$ ee.

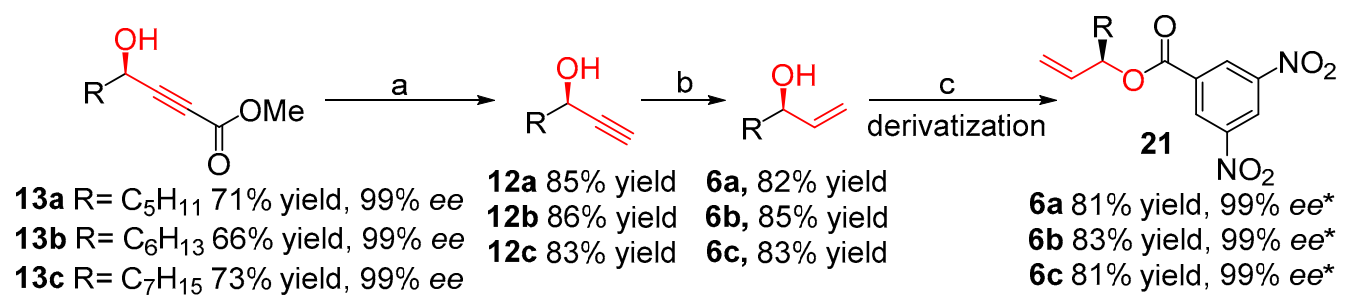

Scheme 3. Total synthesis of (R)-matsutakeol and its natural analogs $6 \mathbf{a}-\mathbf{c}$ by using $(S, S)$-ProPhenol (Scheme 1, Method 3). Reagents and conditions: (a) (i) $\mathrm{LiOH}, \mathrm{THF}, 0 \sim 25^{\circ} \mathrm{C}, 1 \mathrm{~h}$; (ii) $\mathrm{CuCl}, \mathrm{CH}_{3} \mathrm{CN}, 13 \mathrm{~h}$; (b) $\mathrm{Ni}(\mathrm{OAc})_{2}, \mathrm{NaBH}_{4}, \mathrm{EDA}, \mathrm{EtOH}, 0 \sim 25^{\circ} \mathrm{C}$; (c) 3,5-Dinitrobenzoyl chloride, $\mathrm{Et}_{3} \mathrm{~N}, 0 \sim 25^{\circ} \mathrm{C}$, overnight;

* The ee value is determined by HPLC on a chiral column after derivatization.

The BINOL-ZnEt 2 -Ti (IV) complex catalyzed asymmetric addition of trimethylsilylacetylene to the aliphatic aldehydes was well developed by Pu's group [20,22,34]. It is also a practical approach for providing chiral acetylene alcohols. In the optimization procedure, to increase the catalyst loading of $(R, R)$-ProPhenol 16b (entry 1 and 2 ) and to reduce the temperature (entry 2 and 3) could slightly improve the ee value, of product $13 \mathbf{a}^{\prime}$ (Table 2, entry 2, 72\% yield, 78\% ee). Compared to addition catalyzed by $(R, R)$-ProPhenol $\mathbf{1 6 b}$, almost no product was detected at $-10{ }^{\circ} \mathrm{C}$ (Table 2 , entry 4). The reaction yields and optical yields were increased on increasing the amount of BINOL (Table 2, entry 5-7). When the amount of BINOL was increased to $60 \%$, there was no obvious improvement in yield and ee value (entry 7,67\% yield, $81 \%$ ee). Compared to the reaction catalyzed by $(R, R)$-ProPhenol 16b (entry 2, 72\% yield, 78\% ee and entry 3,74\% yield, 76\% ee), the asymmetric addition of trimethylsilylacetylene by using BINOL as chiral ligand could be smoothly carried out at room temperature, affording higher ee value and almost the same yield (entry 6, 71\% yield, 80\% ee).

Table 2. Screening of reaction conditions and ligands of asymmetric addition of $15^{\prime}$ with $\mathbf{1 4 a}$.

\begin{tabular}{|c|c|c|c|c|c|}
\hline $\begin{array}{r}{ }_{5} \mathrm{H}_{11} \\
14 \mathrm{a}\end{array}$ & $15^{\prime}$ & $\frac{(S) \text {-BINC }}{\begin{array}{r}\text { alkyl zir } \\
\text { toluen }\end{array}}$ & 13 & $\mathrm{SiMe}_{3}$ & $17 \mathrm{~b}$ \\
\hline Entry & Ligand & Ligand $/ \%$ & Temp $/{ }^{\circ} \mathrm{C}$ & Yield $/ \%^{c}$ & $e e / \% \mathrm{~d}$ \\
\hline $1^{a}$ & $16 \mathrm{~b}$ & 20 & -10 & 70 & 75 \\
\hline $2^{a}$ & $16 b$ & 40 & -10 & 72 & 78 \\
\hline $3^{a}$ & $16 b$ & 40 & 25 & 74 & 76 \\
\hline $4^{b}$ & $17 \mathrm{~b}$ & 40 & -10 & - & - \\
\hline $5^{b}$ & $17 \mathrm{~b}$ & 20 & 25 & 52 & 70 \\
\hline $6^{b}$ & $17 \mathrm{~b}$ & 40 & 25 & 71 & 80 \\
\hline $7^{b}$ & $17 \mathrm{~b}$ & 60 & 25 & 67 & 81 \\
\hline
\end{tabular}

\footnotetext{
a The reaction was carried out on a $0.5 \mathrm{mmol}$ scale in toluene $(0.5 \mathrm{~mL})$, the organozinc reagent was $\mathrm{Me}_{2} \mathrm{Zn}$;

b The reaction was carried out on a $0.5 \mathrm{mmol}$ scale in $\mathrm{DCM}(6.0 \mathrm{~mL})$, the organozinc reagent was $\mathrm{Et}_{2} \mathrm{Zn}$,

$\mathrm{Et}_{2} \mathrm{Zn}: \mathrm{Ti}\left(\mathrm{O}^{i} \mathrm{Pr}\right)_{4}$ :aldehyde $=4: 1: 1{ }^{\mathrm{c}}$ Isolated yields; ${ }^{\mathrm{d}}$ The $e e$ values were determined by chiral HPLC.
}

Herein, we attempted to synthesize the above natural products $\mathbf{6 a}-\mathbf{c}$ on gram scale, by employing the (S)-BINOL $\mathbf{1 7 b}$ as the catalyst. As shown in Scheme 4, (S)-BINOL efficiently catalyzed the 
asymmetric addition of trimethylsilylacetylene with the corresponding aliphatic aldehydes $\mathbf{1 4 a}-\mathbf{c}$, affording compounds $13 \mathbf{a}^{\prime}-\mathbf{c}^{\prime}$ in moderate yields $(71 \%-72 \%)$ and enantioselectivities $(80 \%-82 \% e e)$, slightly lower than those of compounds $\mathbf{1 3 a}-\mathbf{c}$. $\mathrm{K}_{2} \mathrm{CO}_{3}$-promoted deprotection of trimethyl group of compounds $13 \mathbf{a}^{\prime}-\mathbf{c}^{\prime}$ under mild conditions gave compounds $12 \mathbf{a}-\mathbf{c}$ in good yields (87\%-89\%) [52]. Following the same procedures as shown in Scheme 3, compounds $\mathbf{6 a - c}$ were obtained in moderate yields $(81 \%-83 \%)$ and nearly without loss of enantioselectivities $(79 \%-82 \%$ ee, the $e e$ values were measured by HPLC after derived by 3,5-dinitrobenzoyl chloride). The enantiomeric purity of 21a-c could be improved to over $99 \%$ ee by slow recrystallization from diethylether $/ n$-hexane (1:5) at a low temperature. Subsequently, the esters $21 \mathbf{a}-\mathbf{c}$ could be quantitatively hydrolyzed to the corresponding chiral alcohols $\mathbf{6 a}-\mathbf{c}$ followed in our previous group research [22].

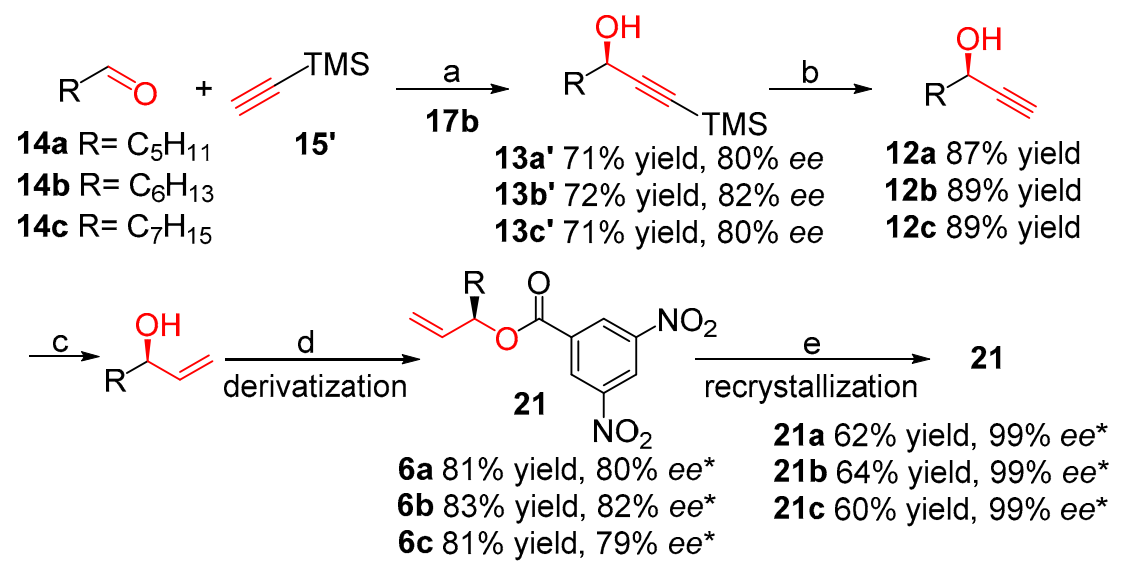

Scheme 4. Total synthesis of (R)-matsutakeol and its natural analogs $6 \mathbf{a}-\mathbf{c}$ by using (S)-BINOL (Scheme 1, Method 3). Reagents and conditions: (a) (i) $\mathrm{ZnEt}_{2}, \mathrm{THF}, \mathrm{N}_{2}, \mathrm{rt}$; (ii) $\mathrm{Ti}\left({ }^{i} \mathrm{PrO}\right)_{4}, \mathrm{THF}, \mathrm{N}_{2}$, rt; (b) $\mathrm{K}_{2} \mathrm{CO}_{3}, \mathrm{MeOH}, 0 \sim 25{ }^{\circ} \mathrm{C}$; (c) Ni(OAc) $2, \mathrm{NaBH}_{4}, \mathrm{EDA}, \mathrm{EtOH}, 0 \sim 25{ }^{\circ} \mathrm{C}$; (d) 3,5-Dinitrobenzoyl chloride, $\mathrm{Et}_{3} \mathrm{~N}, 0 \sim 25{ }^{\circ} \mathrm{C}$, overnight; (e) Recrystallization from diethylether/ $n$-hexane $(1 / 5)$ at $0 \sim 25{ }^{\circ} \mathrm{C}$,

* The $e e$ value is determined by HPLC on a chiral column after derivatization.

\section{Materials and Methods}

All reactions were performed under an argon atmosphere. Solvents were dried according to standard procedures and distilled before use. All reagents were purchased commercially and used without further purification, unless stated otherwise. ${ }^{1} \mathrm{H}$ - and ${ }^{13} \mathrm{C}-\mathrm{NMR}$ spectra were recorded at 300 and $75 \mathrm{MHz}$, respectively. High-resolution mass spectra were recorded on an agilent instrument by the TOF MS technique. Enantiomeric excesses (ee) were determined by chiral HPLC analyses using a chiral column (Chiralpak OD-H, AD-H, OJ-H), and elution with isopropanol-hexane. The optical rotations were measured on PERKIN ELMER 341 Polarimeter. ${ }^{1} \mathrm{H}-,{ }^{13} \mathrm{C}-\mathrm{NMR}$ spectra and HPLC chromatography of the chiral products are in the Supplementary Materials.

\subsection{General Procedure of Asymmetric Addition of Methyl Propiolate to Aliphatic Aldehydes (Table 1)}

To a stirred solution of methyl propiolate ( $84 \mathrm{mg}, 1 \mathrm{mmol}),(S, S)-P r o P h e n o l ~(128 \mathrm{mg}, 0.2 \mathrm{mmol})$, triphenylphosphine oxide $(111 \mathrm{mg}, 0.4 \mathrm{mmol})$ in toluene $(1 \mathrm{~mL})$, dimethylzinc $(2.5 \mathrm{~mL}, 1.2 \mathrm{M}$ in toluene, $3 \mathrm{mmol})$ was added slowly at $-10^{\circ} \mathrm{C}$. After stirring for $1.5 \mathrm{~h}$ at $-10^{\circ} \mathrm{C}$, aldehyde $(1.5 \mathrm{mmol})$ in toluene $(3 \mathrm{~mL})$ was added via syringe at a slow rate in $24 \mathrm{~h}$ at $-10^{\circ} \mathrm{C}$, and quenched with water $(10 \mathrm{~mL})$. The mixture was filtered through a celite pad. The aqueous phase was extracted with ether. The combined organic phases were washed with saturated brine solution, dried over anhydrous $\mathrm{Na}_{2} \mathrm{SO}_{4}$, and concentrated under reduced pressure. The residue was purified by silica gel chromatography to get the product $[25,36]$. 


\subsubsection{Synthesis of (R)-Methyl-4-hydroxynon-2-ynoate: $(R)-13 a$}

Following the general procedure, the residue was purified by silica gel chromatography (hexanes/ethyl acetate 8:1) to offer $(R)-13 \mathrm{a}(131 \mathrm{mg}, 71 \%$ yield, $99 \% e e)$ as colorless oil. $[\alpha]_{\mathrm{D}}^{25}=+5.7$ (c 1.05, $\left.\mathrm{CH}_{2} \mathrm{Cl}_{2}\right),{ }^{1} \mathrm{H}-\mathrm{NMR}\left(300 \mathrm{MHz}, \mathrm{CDCl}_{3}\right) \delta(\mathrm{ppm}): 4.57-4.44(\mathrm{~m}, 1 \mathrm{H}), 3.76(\mathrm{~s}, 3 \mathrm{H}), 2.66(\mathrm{~d}, J=4.7 \mathrm{~Hz}$, $1 \mathrm{H}), 1.80-1.67(\mathrm{~m}, 2 \mathrm{H}), 1.52-1.38(\mathrm{~m}, 2 \mathrm{H}), 1.35-1.25(\mathrm{~m}, 4 \mathrm{H}), 0.88(\mathrm{t}, J=6.7 \mathrm{~Hz}, 3 \mathrm{H}) .{ }^{13} \mathrm{C}-\mathrm{NMR}(75 \mathrm{MHz}$, $\left.\mathrm{CDCl}_{3}\right) \delta$ (ppm): $153.57,88.19,75.78,61.71,52.40,36.46,30.95,24.23,22.08,13.54$. HRMS ESI [M + Na] ${ }^{+}$ calcd for $\mathrm{C}_{10} \mathrm{H}_{16} \mathrm{NaO}_{3}{ }^{+}$207.0992, found 207.0992. Enantiomeric excess was determined by HPLC with a Chiralcel OD-H column (98:2 $n$-hexanes:isopropanol, $1.0 \mathrm{~mL} / \mathrm{min}, 210 \mathrm{~nm})$, major $(R)$-enantiomer $\mathrm{t}_{\mathrm{r}}=14.93 \mathrm{~min}$, minor $(S)$-enantiomer $\mathrm{t}_{\mathrm{r}}=16.73 \mathrm{~min}$.

\subsubsection{Synthesis of $(R)$-Methyl-4-hydroxydec-2-ynoate: $(R)-\mathbf{1 3 b}$}

Following the general procedure, the residue was purified by silica gel chromatography (hexanes/ethyl acetate 8:1) to offer $(R)-\mathbf{1 3 b}(131 \mathrm{mg}, 66 \%$ yield, $99 \% e e)$ as colorless oil. $[\alpha]_{D}^{25}=+5.8$ (c 1.05, $\left.\mathrm{CH}_{2} \mathrm{Cl}_{2}\right),{ }^{1} \mathrm{H}-\mathrm{NMR}\left(300 \mathrm{MHz}, \mathrm{CDCl}_{3}\right) \delta(\mathrm{ppm}): 4.57-4.44(\mathrm{~m}, 1 \mathrm{H}), 3.77(\mathrm{~s}, 3 \mathrm{H}), 2.36(\mathrm{~d}, J=5.7 \mathrm{~Hz}$, $1 \mathrm{H}), 1.84-1.71(\mathrm{~m}, 2 \mathrm{H}), 1.51-1.24(\mathrm{~m}, 8 \mathrm{H}), 0.87(\mathrm{t}, J=6.7 \mathrm{~Hz}, 3 \mathrm{H}) .{ }^{13} \mathrm{C}-\mathrm{NMR}\left(75 \mathrm{MHz}, \mathrm{CDCl}_{3}\right) \delta(\mathrm{ppm})$ : $153.51,88.07,75.85,61.76,52.40,36.53,31.25,28.44,24.51,22.16,13.62$. HRMS ESI $[\mathrm{M}+\mathrm{Na}]^{+}$calcd for $\mathrm{C}_{11} \mathrm{H}_{18} \mathrm{NaO}_{3}{ }^{+} 221.1148$, found 221.1150. Enantiomeric excess was determined by HPLC with a Chiralcel OD-H column (99:1 $n$-hexanes:isopropanol, $0.7 \mathrm{~mL} / \mathrm{min}, 210 \mathrm{~nm}$ ), major $(R)$-enantiomer $\mathrm{t}_{\mathrm{r}}=31.48 \mathrm{~min}$, minor $(S)$-enantiomer $\mathrm{t}_{\mathrm{r}}=37.63 \mathrm{~min}$.

\subsubsection{Synthesis of $(R)-$ Methyl-4-hydroxyundec-2-ynoate: $(R)-13 c$}

Following the general procedure, the residue was purified by silica gel chromatography (hexanes/ethyl acetate 8:1) to offer $(R)-\mathbf{1 3 c}(155 \mathrm{mg}, 73 \%$ yield, $99 \% e e)$ as colorless oil. $[\alpha]_{\mathrm{D}}^{25}=+6.7$ (c 1.05, $\left.\mathrm{CH}_{2} \mathrm{Cl}_{2}\right),{ }^{1} \mathrm{H}-\mathrm{NMR}\left(300 \mathrm{MHz}, \mathrm{CDCl}_{3}\right) \delta(\mathrm{ppm}): 4.57-4.44(\mathrm{~m}, 1 \mathrm{H}), 3.76(\mathrm{~s}, 3 \mathrm{H}), 2.67(\mathrm{~d}$, $J=5.8 \mathrm{~Hz}, 1 \mathrm{H}), 1.76-1.70(\mathrm{~m}, 2 \mathrm{H}), 1.46-1.42(\mathrm{~m}, 2 \mathrm{H}), 1.35-1.23(\mathrm{~m}, 8 \mathrm{H}), 0.92-0.82(\mathrm{~m}, 3 \mathrm{H}) .{ }^{13} \mathrm{C}-\mathrm{NMR}$ $\left(75 \mathrm{MHz}, \mathrm{CDCl}_{3}\right) \delta(\mathrm{ppm}):$ 153.58, 88.22, 75.77, 61.70, 52.41, 36.50, 31.36, 28.75, 28.72, 24.56, 22.23, 13.66. HRMS ESI [M + Na] ${ }^{+}$calcd for $\mathrm{C}_{12} \mathrm{H}_{20} \mathrm{NaO}_{3}{ }^{+} 235.1305$, found 235.1305. Enantiomeric excess was determined by HPLC with a Chiralcel OD-H column (99:1 $\mathrm{n}$-hexanes:isopropanol, $1.0 \mathrm{~mL} / \mathrm{min}$, $210 \mathrm{~nm}$ ), major $(R)$-enantiomer $\mathrm{t}_{\mathrm{r}}=13.97 \mathrm{~min}$, minor $(S)$-enantiomer $\mathrm{t}_{\mathrm{r}}=15.47 \mathrm{~min}$.

\subsubsection{Synthesis of (R)-Methyl-4-hydroxypent-2-ynoate: $(R)-\mathbf{1 3 d}$}

Following the general procedure, the residue was purified by silica gel chromatography (hexanes/ethyl acetate $8: 1)$ to offer $(R)-\mathbf{1 3 d}\left(81 \mathrm{mg}, 63 \%\right.$ yield, $99 \%$ ee) as colorless oil. $[\alpha]_{\mathrm{D}}^{25}=+3.4$ (c 1.05, $\left.\mathrm{CH}_{2} \mathrm{Cl}_{2}\right),{ }^{1} \mathrm{H}-\mathrm{NMR}\left(300 \mathrm{MHz}, \mathrm{CDCl}_{3}\right) \delta(\mathrm{ppm}): 4.57-4.44(\mathrm{~m}, 1 \mathrm{H}), 3.80(\mathrm{~s}, 3 \mathrm{H}), 2.97(\mathrm{~d}, J=5.6 \mathrm{~Hz}$, $1 \mathrm{H}), 1.53(\mathrm{~d}, J=6.7 \mathrm{~Hz}, 3 \mathrm{H}) .{ }^{13} \mathrm{C}-\mathrm{NMR}\left(75 \mathrm{MHz}, \mathrm{CDCl}_{3}\right) \delta(\mathrm{ppm}): 153.61,88.72,74.99,57.53,52.48$, 22.85. HRMS ESI [M + Na] ${ }^{+}$calcd for $\mathrm{C}_{6} \mathrm{H}_{8} \mathrm{NaO}_{3}{ }^{+} 151.0366$, found 151.0365. Enantiomeric excess was determined by HPLC with a Chiralcel OD-H column (98:2 $n$-hexanes:isopropanol, $1.0 \mathrm{~mL} / \mathrm{min}$, $220 \mathrm{~nm}$ ), major $(R)$-enantiomer $t_{\mathrm{r}}=23.06 \mathrm{~min}$, minor $(S)$-enantiomer $\mathrm{t}_{\mathrm{r}}=26.75 \mathrm{~min}$.

\subsubsection{Synthesis of Methyl (R)-Methyl-4-hydroxyhex-2-ynoate: $(R)-\mathbf{1 3 e}$}

Following the general procedure, the residue was purified by silica gel chromatography (hexanes/ethyl acetate 8:1) to offer $(R)-\mathbf{1 3 e}(101 \mathrm{mg}, 71 \%$ yield, $99 \% e e)$ as colorless oil. $[\alpha]_{\mathrm{D}}^{25}=+8.2$ (c 1.05, $\left.\mathrm{CH}_{2} \mathrm{Cl}_{2}\right),{ }^{1} \mathrm{H}-\mathrm{NMR}\left(300 \mathrm{MHz}, \mathrm{CDCl}_{3}\right) \delta(\mathrm{ppm}): 4.57-4.44(\mathrm{~m}, 1 \mathrm{H}), 3.79(\mathrm{~s}, 3 \mathrm{H}), 3.09$ $(\mathrm{d}, J=5.8 \mathrm{~Hz}, 1 \mathrm{H}), 1.87-1.75(\mathrm{~m}, 2 \mathrm{H}), 1.04(\mathrm{t}, J=7.4 \mathrm{~Hz}, 3 \mathrm{H}) .{ }^{13} \mathrm{C}-\mathrm{NMR}\left(75 \mathrm{MHz}, \mathrm{CDCl}_{3}\right) \delta$ (ppm): 153.64, 88.07, 75.76, 62.84, 52.45, 29.64, 8.88. HRMS ESI $[\mathrm{M}+\mathrm{Na}]^{+}$calcd for $\mathrm{C}_{7} \mathrm{H}_{10} \mathrm{NaO}_{3}{ }^{+}$ 165.0522, found 165.0522. Enantiomeric excess was determined by HPLC with a Chiralcel OD-H column (98:2 $n$-hexanes:isopropanol, $1.0 \mathrm{~mL} / \mathrm{min}, 220 \mathrm{~nm}$ ), major $(R)$-enantiomer $\mathrm{t}_{\mathrm{r}}=20.98 \mathrm{~min}$, minor $(S)$-enantiomer $t_{r}=23.86 \mathrm{~min}$. 


\subsubsection{Synthesis of (R)-Methyl-4-hydroxyhept-2-ynoate: $(R)-\mathbf{1 3 f}$}

Following the general procedure, the residue was purified by silica gel chromatography (hexanes/ethyl acetate 8:1) to offer $(R)-13 \mathrm{f}(105 \mathrm{mg}, 67 \%$ yield, $99 \% e e)$ as colorless oil. $[\alpha]_{\mathrm{D}}^{25}=+5.4$ (c 1.05, $\left.\mathrm{CH}_{2} \mathrm{Cl}_{2}\right),{ }^{1} \mathrm{H}-\mathrm{NMR}\left(300 \mathrm{MHz}, \mathrm{CDCl}_{3}\right) \mathcal{\delta}(\mathrm{ppm}): 4.57-4.44(\mathrm{~m}, 1 \mathrm{H}), 3.79(\mathrm{~s}, 3 \mathrm{H}), 3.02(\mathrm{~d}, J=5.8 \mathrm{~Hz}$, $1 \mathrm{H}), 1.83-1.66(\mathrm{~m}, 2 \mathrm{H}), 1.58-1.41(\mathrm{~m}, 2 \mathrm{H}), 0.96(\mathrm{t}, J=7.3 \mathrm{~Hz}, 3 \mathrm{H}) .{ }^{13} \mathrm{C}-\mathrm{NMR}\left(75 \mathrm{MHz}, \mathrm{CDCl}_{3}\right) \delta(\mathrm{ppm}):$ 153.64, 88.29, 75.69, 61.40, 52.45, 38.47, 17.88, 13.22. HRMS ESI $[\mathrm{M}+\mathrm{Na}]^{+}$calcd for $\mathrm{C}_{8} \mathrm{H}_{12} \mathrm{NaO}_{3}{ }^{+}$ 179.0679, found 179.0681. Enantiomeric excess was determined by HPLC with a Chiralcel OD-H column (98:2 n-hexanes:isopropanol, $1.0 \mathrm{~mL} / \mathrm{min}, 220 \mathrm{~nm}$ ), major $(R)$-enantiomer $\mathrm{t}_{\mathrm{r}}=24.14 \mathrm{~min}$, minor $(S)$-enantiomer $t_{r}=25.45 \mathrm{~min}$.

\subsubsection{Synthesis of (R)-Methyl-4-hydroxyoct-2-ynoate: $(R)-\mathbf{1 3 g}$}

Following the general procedure, the residue was purified by silica gel chromatography (hexanes/ethyl acetate 8:1) to offer $(R)-\mathbf{1 3 g}(116 \mathrm{mg}, 68 \%$ yield, $99 \% e e)$ as colorless oil. $[\alpha]_{D}^{25}=+2.3$ (c 1.05, $\left.\mathrm{CH}_{2} \mathrm{Cl}_{2}\right),{ }^{1} \mathrm{H}-\mathrm{NMR}\left(300 \mathrm{MHz}, \mathrm{CDCl}_{3}\right) \delta(\mathrm{ppm}): 4.55-4.44(\mathrm{~m}, 1 \mathrm{H}), 3.77(\mathrm{~s}, 3 \mathrm{H}), 2.39(\mathrm{~d}, J=5.8 \mathrm{~Hz}$, $1 \mathrm{H}), 1.82-1.68(\mathrm{~m}, 2 \mathrm{H}), 1.48-1.31(\mathrm{~m}, 4 \mathrm{H}), 0.91(\mathrm{t}, J=7.1 \mathrm{~Hz}, 3 \mathrm{H}) .{ }^{13} \mathrm{C}-\mathrm{NMR}\left(75 \mathrm{MHz}, \mathrm{CDCl}_{3}\right) \delta(\mathrm{ppm})$ : $153.51,88.06,75.85,61.75,52.40,36.23,26.66,21.88,13.48$. HRMS ESI $[M+\mathrm{Na}]^{+}$calcd for $\mathrm{C}_{9} \mathrm{H}_{14} \mathrm{NaO}_{3}{ }^{+}$ 193.0835, found 193.0835. Enantiomeric excess was determined by HPLC with a Chiralcel OJ-H column (95:5 n-hexanes:isopropanol, $1.0 \mathrm{~mL} / \mathrm{min}, 220 \mathrm{~nm}$ ), major $(R)$-enantiomer $\mathrm{t}_{\mathrm{r}}=7.45 \mathrm{~min}$, minor (S)-enantiomer $\mathrm{t}_{\mathrm{r}}=8.02 \mathrm{~min}$.

\subsubsection{Synthesis of (R)-Methyl-4-hydroxy-5-methylhex-2-ynoate: $(R)-\mathbf{1 3 h}$}

Following the general procedure, the residue was purified by silica gel chromatography (hexanes/ethyl acetate 8:1) to offer $(R)-\mathbf{1 3 h}(111 \mathrm{mg}, 71 \%$ yield, $99 \% e e)$ as colorless oil. $[\alpha]_{\mathrm{D}}^{25}=+7.2$ (c 1.05, $\left.\mathrm{CH}_{2} \mathrm{Cl}_{2}\right),{ }^{1} \mathrm{H}-\mathrm{NMR}\left(300 \mathrm{MHz}, \mathrm{CDCl}_{3}\right) \delta(\mathrm{ppm}): 4.27(\mathrm{t}, J=5.9 \mathrm{~Hz}, 1 \mathrm{H}), 3.76(\mathrm{~s}, 3 \mathrm{H}), 3.11$ $(\mathrm{d}, J=5.9 \mathrm{~Hz}, 1 \mathrm{H}), 1.97-1.90(\mathrm{~m}, 1 \mathrm{H}), 1.00(\mathrm{dd}, J=6.8,4.4 \mathrm{~Hz}, 6 \mathrm{H}) .{ }^{13} \mathrm{C}-\mathrm{NMR}\left(75 \mathrm{MHz}, \mathrm{CDCl}_{3}\right) \delta$ (ppm): 153.64, 87.36, 67.05, 52.44, 33.79, 17.58, 17.09. HRMS ESI $[\mathrm{M}+\mathrm{Na}]^{+}$calcd for $\mathrm{C}_{8} \mathrm{H}_{12} \mathrm{NaO}_{3}{ }^{+}$ 179.0679, found 179.0680. Enantiomeric excess was determined by HPLC with a Chiralcel OJ-H column (95:5 $n$-hexanes:isopropanol, $1.0 \mathrm{~mL} / \mathrm{min}, 220 \mathrm{~nm}$ ), major $(R)$-enantiomer $\mathrm{t}_{\mathrm{r}}=8.66 \mathrm{~min}$, minor $(S)$-enantiomer $t_{r}=10.71 \mathrm{~min}$.

\subsubsection{Synthesis of $(R)-$ Methyl-4-hydroxyoct-7-en-2-ynoate: $(R)-\mathbf{1 3 i}$}

Following the general procedure, the residue was purified by silica gel chromatography (hexanes/ethyl acetate 8:1) to offer $(R)-\mathbf{1 3 i}(114 \mathrm{mg}, 68 \%$ yield, $97 \%$ ee $)$ as colorless oil. $[\alpha]_{\mathrm{D}}^{25}=-10.1$ (c 1.05, $\left.\mathrm{CH}_{2} \mathrm{Cl}_{2}\right),{ }^{1} \mathrm{H}-\mathrm{NMR}\left(300 \mathrm{MHz}, \mathrm{CDCl}_{3}\right) \delta(\mathrm{ppm})$ : 5.89-5.75 (m, 1H), 5.12-4.96 (m, 2H), $4.52(\mathrm{q}$, $J=6.4 \mathrm{~Hz}, 1 \mathrm{H}), 3.79(\mathrm{~s}, 3 \mathrm{H}), 2.64(\mathrm{~d}, J=5.5 \mathrm{~Hz}, 1 \mathrm{H}), 2.26(\mathrm{dd}, J=14.4,6.8 \mathrm{~Hz}, 2 \mathrm{H}), 1.92-1.81(\mathrm{~m}, 2 \mathrm{H})$. ${ }^{13} \mathrm{C}-\mathrm{NMR}\left(75 \mathrm{MHz}, \mathrm{CDCl}_{3}\right) \delta(\mathrm{ppm}): 153.53,136.64,115.46,87.75,76.03,61.08,52.48,35.48,28.73$. HRMS ESI $[\mathrm{M}+\mathrm{Na}]^{+}$calcd for $\mathrm{C}_{9} \mathrm{H}_{12} \mathrm{NaO}_{3}{ }^{+} 191.0679$, found 191.0679. Enantiomeric excess was determined by HPLC with a Chiralcel OJ-H column (95:5 $n$-hexanes:isopropanol, $1.0 \mathrm{~mL} / \mathrm{min}, 220 \mathrm{~nm}$ ), major $(R)$-enantiomer $t_{\mathrm{r}}=9.01 \mathrm{~min}$, minor $(S)$-enantiomer $t_{\mathrm{r}}=9.75 \mathrm{~min}$.

\subsection{General Procedure for the Synthesis of Chiral Alkynols $\mathbf{1 2}$ from Propargyl Alcohols $\mathbf{1 3}$}

A solution of the chiral alkynol $(5 \mathrm{mmol})$ and THF $(60 \mathrm{~mL})$ were cooled to $0{ }^{\circ} \mathrm{C}, 1 \mathrm{M}$ aq $\mathrm{LiOH}$ ( $25 \mathrm{mmol}, 5 \mathrm{eq}$ ) was added at a slow rate. The solution was warmed to rt and stirred for an additional $1 \mathrm{~h}$ before it was quenched with $1 \mathrm{M}^{2} \mathrm{NaHSO}_{4}(50 \mathrm{~mL})$. The aqueous phase was extracted by ethyl acetate. The combined organic phases were dried over anhydrous $\mathrm{Na}_{2} \mathrm{SO}_{4}$, and concentrated under reduced pressure. The residue was dissolved in acetonitrile $(12 \mathrm{~mL}), \mathrm{CuCl}(0.5940 \mathrm{~g}, 6 \mathrm{mmol}, 1.2 \mathrm{eq})$ was added in one portion to the mixture. The mixture was allowed to warm to r.t. and stirred for another $13 \mathrm{~h}$. The aqueous phase was extracted by ether. The combined organic phases were dried 
over anhydrous $\mathrm{Na}_{2} \mathrm{SO}_{4}$, and concentrated under reduced pressure. The residue was purified by silica gel chromatography to get the product.

\subsubsection{Synthesis of (R)-Oct-1-yn-3-ol: (R)-12a}

Following the general procedure, the residue was purified by silica gel chromatography (hexanes/ethyl acetate 15:1) to offer $(R)-\mathbf{1 2 a}\left(536 \mathrm{mg}\right.$, 85\% yield) as colorless oil. $[\alpha]_{\mathrm{D}}^{25}=+18.5$ (c 1.0, ethyl ether), lit. [53] $[\alpha]_{\mathrm{D}}^{25}=+19.3$ (c 1.0, ethyl ether), ${ }^{1} \mathrm{H}-\mathrm{NMR}\left(300 \mathrm{MHz}, \mathrm{CDCl}_{3}\right) \delta(\mathrm{ppm}): 4.33-4.27$ $(\mathrm{m}, 1 \mathrm{H}), 3.10(\mathrm{~s}, 1 \mathrm{H}), 2.40(\mathrm{~d}, J=2.1 \mathrm{~Hz}, 1 \mathrm{H}), 1.69-1.60(\mathrm{~m}, 2 \mathrm{H}), 1.46-1.33(\mathrm{~m}, 2 \mathrm{H}), 1.33-1.11(\mathrm{~m}, 4 \mathrm{H})$, $0.83(\mathrm{t}, J=7.0 \mathrm{~Hz}, 3 \mathrm{H}) .{ }^{13} \mathrm{C}-\mathrm{NMR}\left(75 \mathrm{MHz}, \mathrm{CDCl}_{3}\right) \mathcal{\delta}(\mathrm{ppm}): 84.82,72.31,61.71,37.15,31.03,24.33$, 22.11, 13.53. HRMS ESI [M + Na] ${ }^{+}$calcd for $\mathrm{C}_{8} \mathrm{H}_{14} \mathrm{NaO}^{+} 149.0937$, found 149.0938.

\subsubsection{Synthesis of $(R)-$ Non-1-yn-3-ol: $(R)-\mathbf{1 2 b}$}

Following the general procedure, the residue was purified by silica gel chromatography (hexanes/ethyl acetate 15:1) to offer $(R)-\mathbf{1 2 b}\left(603 \mathrm{mg}, 86 \%\right.$ yield) as colorless oil. $[\alpha]_{\mathrm{D}}^{25}=+5.3(c 2.0$, $\left.\mathrm{CHCl}_{3}\right)$, lit. [54] $[\alpha]_{\mathrm{D}}^{25}=+4.6\left(c 1.92, \mathrm{CHCl}_{3}\right),{ }^{1} \mathrm{H}-\mathrm{NMR}\left(300 \mathrm{MHz}, \mathrm{CDCl}_{3}\right) \delta(\mathrm{ppm}): 4.37(\mathrm{t}, J=5.9 \mathrm{~Hz}$, $1 \mathrm{H}), 2.47(\mathrm{~d}, J=5.9 \mathrm{~Hz}, 1 \mathrm{H}), 2.38(\mathrm{~s}, 1 \mathrm{H}), 1.75-1.68(\mathrm{~m}, 2 \mathrm{H}), 1.52-1.41(\mathrm{~m}, 2 \mathrm{H}), 1.38-1.27(\mathrm{~m}, 6 \mathrm{H})$, $0.89(\mathrm{t}, J=6.7 \mathrm{~Hz}, 3 \mathrm{H}) .{ }^{13} \mathrm{C}-\mathrm{NMR}\left(75 \mathrm{MHz}, \mathrm{CDCl}_{3}\right) \delta(\mathrm{ppm}): 84.78,72.38,61.90,37.28,31.35,28.53$, 24.62, 22.19, 13.66. HRMS ESI [M + Na] ${ }^{+}$calcd for $\mathrm{C}_{9} \mathrm{H}_{16} \mathrm{NaO}^{+}$Exact Mass: 163.1093, found 163.1093.

\subsubsection{Synthesis of (R)-Dec-1-yn-3-ol: $(R)-\mathbf{1 2 c}$}

Following the general procedure, the residue was purified by silica gel chromatography (hexanes/ethyl acetate 15:1) to offer $(R)-12 \mathrm{c}(640 \mathrm{mg}, 83 \% \text { yield) as colorless oil. [ } \alpha]_{\mathrm{D}}^{25}=+6.2(c 1.1$, $\left.\mathrm{CHCl}_{3}\right)$, lit. [55] $[\alpha]_{\mathrm{D}}^{25}=+4.2\left(c 1.1, \mathrm{CHCl}_{3}\right),{ }^{1} \mathrm{H}-\mathrm{NMR}\left(300 \mathrm{MHz}, \mathrm{CDCl}_{3}\right) \delta(\mathrm{ppm}): 4.35(\mathrm{dd}, J=6.5$, $4.9 \mathrm{~Hz}, 1 \mathrm{H}), 2.44(\mathrm{~d}, J=2.1 \mathrm{~Hz}, 1 \mathrm{H}), 2.33(\mathrm{~s}, 1 \mathrm{H}), 1.78-1.60(\mathrm{~m}, 2 \mathrm{H}), 1.50-1.38(\mathrm{~m}, 2 \mathrm{H}), 1.32-1.26(\mathrm{~m}, 8 \mathrm{H})$, $0.86(\mathrm{t}, J=6.8 \mathrm{~Hz}, 3 \mathrm{H}) .{ }^{13} \mathrm{C}-\mathrm{NMR}\left(75 \mathrm{MHz}, \mathrm{CDCl}_{3}\right) \delta(\mathrm{ppm}): 84.74,72.41,61.97,37.31,31.40,29.34$, $28.83,24.66,22.26,13.69$. HRMS ESI [M + Na] $]^{+}$calcd for $\mathrm{C}_{10} \mathrm{H}_{18} \mathrm{NaO}^{+} 177.1250$, found 177.1250.

\subsection{General Procedure for the Selective Reduction of the Chiral Alkynols $\mathbf{1 2}$}

To a stirred solution of nickel acetate tetrahydrate $(352 \mathrm{mg}, 2 \mathrm{mmol})$ in ethanol $(5 \mathrm{~mL})$ under hydrogen, sodium borohydride $(76 \mathrm{mg}, 2 \mathrm{mmol})$ in ethanol $(2 \mathrm{~mL})$ was added at $0{ }^{\circ} \mathrm{C}$. After stirring for $1 \mathrm{~h}$ at $25^{\circ} \mathrm{C}$, ethylenediamine $(481 \mathrm{mg}, 8 \mathrm{mmol})$ was added. The reaction mixture was stirred for another $10 \mathrm{~min}$ before chiral alkynol $(2 \mathrm{mmol})$ in ethanol $(2 \mathrm{~mL})$ was added slowly to the reaction mixture at $0{ }^{\circ} \mathrm{C}$. The reaction was allowed to proceed at $25^{\circ} \mathrm{C}$ under hydrogen for $6 \mathrm{~h}$ at $25^{\circ} \mathrm{C}$. The mixture was filtered through a celite pad, diluted with ether, and concentrated under reduced pressure. The residue was purified by silica gel chromatography to get the product.

\subsubsection{Synthesis of $(R)-O c t-1-e n-3-o l: ~(R)-6 a$}

Following the general procedure, the residue was purified by silica gel chromatography (hexanes / ethyl acetate 10:1) to offer $(R)-6 a\left(210 \mathrm{mg}, 82 \%\right.$ yield) as colorless oil. $[\alpha]_{\mathrm{D}}^{25}=-10.8(c 1.1$, $\left.\mathrm{CHCl}_{3}\right)$, lit. [56] $[\alpha]_{\mathrm{D}}^{25}=-10.0\left(c 1.67, \mathrm{CHCl}_{3}\right),{ }^{1} \mathrm{H}-\mathrm{NMR}\left(300 \mathrm{MHz}, \mathrm{CDCl}_{3}\right) \delta(\mathrm{ppm}): 5.95-5.83(\mathrm{~m}, 1 \mathrm{H})$, $5.27-5.21(\mathrm{~m}, 1 \mathrm{H}), 5.14-5.10(\mathrm{~m}, 1 \mathrm{H}), 4.12(\mathrm{~d}, J=4.8 \mathrm{~Hz}, 1 \mathrm{H}), 1.61(\mathrm{~d}, J=2.8 \mathrm{~Hz}, 1 \mathrm{H}), 1.58-1.48(\mathrm{~m}, 2 \mathrm{H})$, $1.44-1.25(\mathrm{~m}, 6 \mathrm{H}), 0.91(\mathrm{t}, J=6.7 \mathrm{~Hz}, 3 \mathrm{H}) .{ }^{13} \mathrm{C}-\mathrm{NMR}\left(75 \mathrm{MHz}, \mathrm{CDCl}_{3}\right) \delta(\mathrm{ppm}): 141.02,114.12,72.92$, $36.68,31.40,24.64,22.23,13.64$. HRMS ESI $[\mathrm{M}+\mathrm{Na}]^{+}$calcd for $\mathrm{C}_{8} \mathrm{H}_{16} \mathrm{NaO}^{+} 151.1093$, found 151.1093.

\subsubsection{Synthesis of $(R)-$ Non-1-en-3-ol: $(R)-6 \mathbf{b}$}

Following the general procedure, the residue was purified by silica gel chromatography (hexanes / ethyl acetate 10:1) to offer $(R)-6 \mathbf{b}\left(242 \mathrm{mg}, 85 \%\right.$ yield) as colorless oil. $[\alpha]_{\mathrm{D}}^{25}=-11.5(c 1.1$, ethanol), lit. [57] $[\alpha]_{\mathrm{D}}^{25}=-13.4$ (c 1.12, ethanol), ${ }^{1} \mathrm{H}-\mathrm{NMR}\left(300 \mathrm{MHz}, \mathrm{CDCl}_{3}\right) \delta$ (ppm): 5.94-5.83 $(\mathrm{m}, 1 \mathrm{H}), 5.27-5.20(\mathrm{~m}, 1 \mathrm{H}), 5.14-5.09(\mathrm{~m}, 1 \mathrm{H}), 4.12(\mathrm{~d}, J=5.9 \mathrm{~Hz}, 1 \mathrm{H}), 1.67(\mathrm{~d}, J=3.5 \mathrm{~Hz}, 1 \mathrm{H}), 1.60-1.49$ 
(m, 2H), 1.40-1.29 (m, 8H), $0.90(\mathrm{t}, J=6.7 \mathrm{~Hz}, 3 \mathrm{H}) .{ }^{13} \mathrm{C}-\mathrm{NMR}\left(75 \mathrm{MHz}, \mathrm{CDCl}_{3}\right) \delta(\mathrm{ppm}): 141.03,114.10$, 72.91, 36.72, 31.43, 28.86, 24.93, 22.22, 13.68. HRMS ESI $[\mathrm{M}+\mathrm{Na}]^{+}$calcd for $\mathrm{C}_{9} \mathrm{H}_{18} \mathrm{NaO}^{+} 165.1250$, found 165.1250 .

\subsubsection{Synthesis of $(R)-D e c-1-e n-3-o l: ~(R)-6 c$}

Following the general procedure, the residue was purified by silica gel chromatography (hexanes/ethyl acetate 10:1) to offer $(R)-6 \mathrm{c}\left(259 \mathrm{mg}, 83 \%\right.$ yield) as colorless oil. $[\alpha]_{\mathrm{D}}^{25}=-16.7(c 1.1$, $\left.\mathrm{CHCl}_{3}\right)$, lit. [58] $[\alpha]_{\mathrm{D}}^{25}=-18.1\left(c 1.22, \mathrm{CHCl}_{3}\right),{ }^{1} \mathrm{H}-\mathrm{NMR}\left(300 \mathrm{MHz}, \mathrm{CDCl}_{3}\right) \delta$ (ppm): 5.92-5.81 (m, 1H), 5.32-5.17 (m, 1H), 5.16-5.06 (m, 1H), 4.15-4.05 (m, 1H), $1.91(\mathrm{~d}, J=3.7 \mathrm{~Hz}, 1 \mathrm{H}), 1.58-1.46(\mathrm{~m}, 2 \mathrm{H})$, 1.40-1.22 (m, 10H), $0.89(\mathrm{t}, J=6.7 \mathrm{~Hz}, 3 \mathrm{H}) .{ }^{13} \mathrm{C}-\mathrm{NMR}\left(75 \mathrm{MHz}, \mathrm{CDCl}_{3}\right) \delta(\mathrm{ppm}): 141.04,114.04,72.86$, $36.70,31.45,29.16,28.88,24.97,22.27,13.68$. HRMS ESI $[\mathrm{M}+\mathrm{Na}]^{+}$calcd for $\mathrm{C}_{10} \mathrm{H}_{20} \mathrm{NaO}^{+} 179.1406$, found 179.1408 .

\subsection{General Procedure for the Esterification Reaction and Determination of Enantiomeric Excess by HPLC}

Triethylamine (152 mg, $1.5 \mathrm{mmol}$ ) and 3,5-dinitrobenzoyl chloride (277 mg, $1.2 \mathrm{mmol}$ ) were added to a stirred solution of the chiral alcohol $(1 \mathrm{mmol})$ in $\mathrm{CH}_{2} \mathrm{Cl}_{2}(6 \mathrm{~mL})$ at $-5{ }^{\circ} \mathrm{C}$. The mixture was stirred for $5 \mathrm{~h}$ at $25^{\circ} \mathrm{C}$ before water $(2 \mathrm{~mL})$ was poured into the mixture at $0{ }^{\circ} \mathrm{C}$. The aqueous phase was extracted with ether, and combined organic phases were washed with saturated brine solution, dried over anhydrous $\mathrm{Na}_{2} \mathrm{SO}_{4}$, and concentrated under reduced pressure. The residue was purified by silica gel chromatography to get the product.

\subsubsection{Synthesis of $(R)-$ Oct-1-en-3-yl-3,5-dinitrobenzoate: $(R)-21 a$}

Following the general procedure, the residue was purified by silica gel chromatography (hexanes/ethyl acetate 8:1) to offer $(R)-21 a\left(306 \mathrm{mg}, 95 \%\right.$ yield, 99\% ee) as white solid. $[\alpha]_{\mathrm{D}}^{25}=-16.2$ (c 1.1, $\left.\mathrm{CH}_{2} \mathrm{Cl}_{2}\right),{ }^{1} \mathrm{H}-\mathrm{NMR}\left(300 \mathrm{MHz}, \mathrm{CDCl}_{3}\right) \delta(\mathrm{ppm}): 9.22(\mathrm{t}, J=2.1 \mathrm{~Hz}, 1 \mathrm{H}), 9.16(\mathrm{~d}, J=2.1 \mathrm{~Hz}$, $2 \mathrm{H}), 5.92(\mathrm{~m} \mathrm{1H}), 5.56(\mathrm{q}, J=6.9 \mathrm{~Hz}, 1 \mathrm{H}), 5.36(\mathrm{~m}, 2 \mathrm{H}), 1.96-1.72(\mathrm{~m}, 2 \mathrm{H}), 1.48-1.26(\mathrm{~m}, 6 \mathrm{H}), 0.89(\mathrm{t}$, $J=6.9 \mathrm{~Hz}, 3 \mathrm{H}) .{ }^{13} \mathrm{C}-\mathrm{NMR}\left(75 \mathrm{MHz}, \mathrm{CDCl}_{3}\right) \delta(\mathrm{ppm}): 161.45,148.34,135.09,134.00,129.02,121.92$, 118.05, 77.82, 33.69, 31.09, 24.40, 22.10, 13.57. HRMS ESI $[\mathrm{M}+\mathrm{Na}]^{+}$calcd for $\mathrm{C}_{15} \mathrm{H}_{18} \mathrm{~N}_{2} \mathrm{NaO}_{6}{ }^{+}$ 345.1057, found 345.1057. Enantiomeric excess was determined by HPLC with a Chiralcel OD-H column (99:1 n-hexanes: isopropanol, $1.0 \mathrm{~mL} / \mathrm{min}, 210 \mathrm{~nm})$, major $(R)$-enantiomer $\mathrm{t}_{\mathrm{r}}=17.74 \mathrm{~min}$, minor $(S)$-enantiomer $\mathrm{t}_{\mathrm{r}}=14.46 \mathrm{~min}$.

\subsubsection{Synthesis of $(R)-N o n-1-e n-3-y 1-3,5-$ dinitrobenzoate: $(R)-\mathbf{2 1 b}$}

Following the general procedure, the residue was purified by silica gel chromatography (hexanes/ethyl acetate 8:1) to offer $(R)-21 b\left(312 \mathrm{mg}, 93 \%\right.$ yield, 99\% ee) as colorless oil. $[\alpha]_{\mathrm{D}}^{25}=-18.3$ (c 1.1, $\left.\mathrm{CH}_{2} \mathrm{Cl}_{2}\right),{ }^{1} \mathrm{H}-\mathrm{NMR}\left(300 \mathrm{MHz}, \mathrm{CDCl}_{3}\right) \delta(\mathrm{ppm}): 9.21(\mathrm{t}, J=2.1 \mathrm{~Hz}, 1 \mathrm{H}), 9.15(\mathrm{~d}, J=2.1 \mathrm{~Hz}$, $2 \mathrm{H}), 6.01-5.84(\mathrm{~m}, 1 \mathrm{H}), 5.56(\mathrm{~d}, J=6.6 \mathrm{~Hz}, 1 \mathrm{H}), 5.34(\mathrm{dd}, J=26.1,13.8 \mathrm{~Hz}, 2 \mathrm{H}), 1.95-1.70(\mathrm{~m}, 2 \mathrm{H})$, 1.45-1.26 (m, 8H), $0.87(\mathrm{t}, J=6.7 \mathrm{~Hz}, 3 \mathrm{H}) .{ }^{13} \mathrm{C}-\mathrm{NMR}\left(75 \mathrm{MHz}, \mathrm{CDCl}_{3}\right) \delta(\mathrm{ppm}): 161.45,148.33,135.11$, $133.99,129.02,121.92,118.01,77.81,33.73,31.27,28.59,24.70,22.17,13.63$. HRMS ESI [M + Na $]^{+}$calcd $^{2}$ for $\mathrm{C}_{16} \mathrm{H}_{20} \mathrm{~N}_{2} \mathrm{NaO}_{6}{ }^{+}$359.1214, found 359.1214. Enantiomeric excess was determined by HPLC with a Chiralcel OD-H column (98:2 n-hexanes:isopropanol, $1.0 \mathrm{~mL} / \mathrm{min}, 210 \mathrm{~nm})$, major $(R)$-enantiomer $\mathrm{t}_{\mathrm{r}}=15.27 \mathrm{~min}$, minor $(S)$-enantiomer $\mathrm{t}_{\mathrm{r}}=12.67 \mathrm{~min}$.

\subsubsection{Synthesis of $(R)-$ Dec-1-en-3-yl-3,5-dinitrobenzoate: $(R)-21 \mathrm{c}$}

Following the general procedure, the residue was purified by silica gel chromatography (hexanes/ethyl acetate 8:1) to offer $(R)-6 \mathrm{c}\left(332 \mathrm{mg}, 95 \%\right.$ yield, 99\% ee) as white solid. $[\alpha]_{\mathrm{D}}^{25}=-21.2$ (c 1.05, $\left.\mathrm{CH}_{2} \mathrm{Cl}_{2}\right),{ }^{1} \mathrm{H}-\mathrm{NMR}\left(300 \mathrm{MHz}, \mathrm{CDCl}_{3}\right) \delta(\mathrm{ppm}): 9.23(\mathrm{t}, J=2.1 \mathrm{~Hz}, 1 \mathrm{H}), 9.16(\mathrm{~d}, J=2.1 \mathrm{~Hz}, 2 \mathrm{H})$, $5.98-5.87(\mathrm{~m}, 1 \mathrm{H}), 5.57(\mathrm{q}, J=6.8 \mathrm{~Hz}, 1 \mathrm{H}), 5.35(\mathrm{dd}, J=25.1,13.8 \mathrm{~Hz}, 2 \mathrm{H}), 1.99-1.71(\mathrm{~m}, 2 \mathrm{H}), 1.40-1.28$ $(\mathrm{m}, 10 \mathrm{H}), 0.88(\mathrm{t}, J=6.6 \mathrm{~Hz}, 3 \mathrm{H}) .{ }^{13} \mathrm{C}-\mathrm{NMR}\left(75 \mathrm{MHz}, \mathrm{CDCl}_{3}\right) \delta(\mathrm{ppm}): 161.45,148.35,135.09,134.02$, 
129.03, 121.92, 118.08, 77.85, 33.74, 31.37, 28.90, 28.75, 24.76, 22.24, 13.68. HRMS ESI [M + Na] ${ }^{+}$calcd for $\mathrm{C}_{17} \mathrm{H}_{22} \mathrm{~N}_{2} \mathrm{NaO}_{6}{ }^{+}$373.1370, found 373.1370. Enantiomeric excess was determined by HPLC with a Chiralcel OD-H column (98:2 $n$-hexanes:isopropanol, $1.0 \mathrm{~mL} / \mathrm{min}, 210 \mathrm{~nm}$ ), major ( $R$ )-enantiomer $\mathrm{t}_{\mathrm{r}}=16.89 \mathrm{~min}$, minor $(S)$-enantiomer $\mathrm{t}_{\mathrm{r}}=13.50 \mathrm{~min}$.

\subsection{General Procedure of Asymmetric Addition of Ethynyltrimethylsilane to Aliphatic Aldehydes}

To a stirred solution of ethynyltrimethylsilane (3922 mg, $40 \mathrm{mmol})$, (S)-BINOL (1144 mg, $4 \mathrm{mmol}$ ), HMPA ( $3584 \mathrm{mg}, 20 \mathrm{mmol}$ ) in methylene chloride $(120 \mathrm{~mL})$, diethylzinc $(40 \mathrm{~mL}, 40 \mathrm{mmol})$ was added slowly at $0{ }^{\circ} \mathrm{C}$. After stirring for $16 \mathrm{~h}$ at $25^{\circ} \mathrm{C}$, Titanium(IV) isopropoxide $(2842 \mathrm{mg}, 10 \mathrm{mmol})$ was added and the stirring was continued for another $1 \mathrm{~h}$ at $25^{\circ} \mathrm{C}$. Then an aldehyde (10 mmol) was added and the reaction was allowed to proceed at $25^{\circ} \mathrm{C}$ for another $6 \mathrm{~h}$ before being quenched with water $(20 \mathrm{~mL})$. The mixture was filtered through a celite pad. The aqueous phase was extracted with ether. The combined organic phases were washed with saturated brine, dried over anhydrous $\mathrm{Na}_{2} \mathrm{SO}_{4}$, and concentrated under reduced pressure. The residue was purified by silica gel chromatography to get the product $[34,40]$.

\subsubsection{Synthesis of $(R)-1$-(Trimethylsilyl)oct-1-yn-3-ol: $(R)-13 \mathbf{a}^{\prime}$}

Following the general procedure, the residue was purified by silica gel chromatography (hexanes/ethyl acetate 20:1) to offer $(R)-\mathbf{1 3} \mathbf{a}^{\prime}(1408 \mathrm{mg}, 71 \%$ yield, $80 \% e e)$ as colorless oil. $[\alpha]_{\mathrm{D}}^{25}=+8.2$ $\left(c 1.1, \mathrm{CHCl}_{3}\right)$, lit. [56] $[\alpha]_{\mathrm{D}}^{25}=+13.6\left(c 1.1, \mathrm{CHCl}_{3}\right),{ }^{1} \mathrm{H}-\mathrm{NMR}\left(300 \mathrm{MHz}, \mathrm{CDCl}_{3}\right) \delta$ (ppm): 4.38 (dd, $J=12.2,6.4 \mathrm{~Hz}, 1 \mathrm{H}), 1.80(\mathrm{~d}, J=5.5 \mathrm{~Hz}, 1 \mathrm{H}), 1.76-1.68(\mathrm{~m}, 2 \mathrm{H}), 1.47(\mathrm{~d}, J=7.3 \mathrm{~Hz}, 2 \mathrm{H}), 1.35(\mathrm{dd}$, $J=7.1,3.5 \mathrm{~Hz}, 4 \mathrm{H}), 0.92(\mathrm{t}, J=6.7 \mathrm{~Hz}, 3 \mathrm{H}), 0.20(\mathrm{~s}, 9 \mathrm{H}) .{ }^{13} \mathrm{C}-\mathrm{NMR}\left(75 \mathrm{MHz}, \mathrm{CDCl}_{3}\right) \delta(\mathrm{ppm}): 106.61$, 88.96, 62.61, 37.35, 31.04, 24.41, 22.16, 13.59, -0.47 . HRMS ESI $[\mathrm{M}+\mathrm{Na}]^{+}$calcd for $\mathrm{C}_{11} \mathrm{H}_{22} \mathrm{NaOSi}^{+}$ 221.1332, found 221.1332. Enantiomeric excess was determined by HPLC with a Chiralcel AD-H column (99:1 n-hexanes:isopropanol, $1.0 \mathrm{~mL} / \mathrm{min}, 210 \mathrm{~nm})$, major $(R)$-enantiomer $\mathrm{t}_{\mathrm{r}}=14.72 \mathrm{~min}$, minor $(S)$-enantiomer $t_{r}=13.52 \mathrm{~min}$.

\subsubsection{Synthesis of $(R)-1-\left(\right.$ Trimethylsilyl)non-1-yn-3-ol: $(R)-13 \mathbf{b}^{\prime}$}

Following the general procedure, the residue was purified by silica gel chromatography (hexanes/ethyl acetate 20:1) to offer $(R)-\mathbf{1 3 b}^{\prime}(1529 \mathrm{mg}, 72 \%$ yield, $82 \% e e)$ as colorless oil. $[\boldsymbol{\alpha}]_{\mathrm{D}}^{25}=+2.3$ (c 1.1, $\left.\mathrm{CHCl}_{3}\right),{ }^{1} \mathrm{H}-\mathrm{NMR}\left(300 \mathrm{MHz}, \mathrm{CDCl}_{3}\right) \mathcal{\delta}(\mathrm{ppm}): 4.38(\mathrm{dd}, J=12.3,6.5 \mathrm{~Hz}, 1 \mathrm{H}), 1.78-1.68(\mathrm{~m}, 3 \mathrm{H})$, $1.46(\mathrm{~d}, J=6.5 \mathrm{~Hz}, 2 \mathrm{H}), 1.38-1.27(\mathrm{~m}, 6 \mathrm{H}), 0.92(\mathrm{t}, J=6.7 \mathrm{~Hz}, 3 \mathrm{H}), 0.20(\mathrm{~s}, 9 \mathrm{H}) .{ }^{13} \mathrm{C}-\mathrm{NMR}\left(75 \mathrm{MHz}, \mathrm{CDCl}_{3}\right)$ $\delta$ (ppm): 106.68, 88.89, 62.54, 37.36, 31.34, 28.52, 24.70, 22.17, 13.67, -0.48 . HRMS ESI [M + Na] ${ }^{+}$calcd for $\mathrm{C}_{12} \mathrm{H}_{24} \mathrm{NaOSi}^{+} 235.1489,235.1490$. Enantiomeric excess was determined by HPLC with a Chiralcel AD-H column (98:2 $n$-hexanes:isopropanol, $1.0 \mathrm{~mL} / \mathrm{min}, 210 \mathrm{~nm})$, major $(R)$-enantiomer $\mathrm{t}_{\mathrm{r}}=13.68 \mathrm{~min}$, minor $(S)$-enantiomer $t_{r}=12.42 \mathrm{~min}$.

\subsubsection{Synthesis of $(R)-1$-(Trimethylsilyl)dec-1-yn-3-ol: $(R)-13 \mathbf{c}^{\prime}$}

Following the general procedure, the residue was purified by silica gel chromatography (hexanes/ethyl acetate 20:1) to offer $(R)-13 \mathbf{c}^{\prime}(1608 \mathrm{mg}, 71 \%$ yield, $80 \% e e)$ as colorless oil. $[\alpha]_{D}^{25}=+3.2$ (c 1.1, $\left.\mathrm{CHCl}_{3}\right) .{ }^{1} \mathrm{H}-\mathrm{NMR}\left(300 \mathrm{MHz}, \mathrm{CDCl}_{3}\right) \delta(\mathrm{ppm}): 4.36(\mathrm{dd}, J=12.3,6.5 \mathrm{~Hz}, 1 \mathrm{H}), 2.00(\mathrm{~m}, 1 \mathrm{H})$, 1.83-1.61 (m, 2H), 1.53-1.40 (m, 2H), 1.40-1.31 (m, 8H), $0.90(\mathrm{t}, J=6.7 \mathrm{~Hz}, 3 \mathrm{H}), 0.18(\mathrm{~m}, 9 \mathrm{H}) .{ }^{13} \mathrm{C}-\mathrm{NMR}$ $\left(75 \mathrm{MHz}, \mathrm{CDCl}_{3}\right) \mathcal{\delta}(\mathrm{ppm}): 106.70,88.87,62.54,37.36,31.39,28.81,28.79,24.74,22.26,13.70,-0.48$. HRMS ESI [M + Na] ${ }^{+}$calcd for $\mathrm{C}_{13} \mathrm{H}_{26} \mathrm{NaOSi}^{+} 249.1645$, found 249.1645. Enantiomeric excess was determined by HPLC with a Chiralcel AD-H column (99:1 $n$-hexanes:isopropanol, $0.9 \mathrm{~mL} / \mathrm{min}, 210 \mathrm{~nm}$ ), major $(R)$-enantiomer $t_{\mathrm{r}}=18.90 \mathrm{~min}$, minor $(S)$-enantiomer $\mathrm{t}_{\mathrm{r}}=16.94 \mathrm{~min}$. 


\subsection{General Procedure for the Synthesis of chIral Alkynols $\mathbf{1 2}$ from Propargyl Alcohols $\mathbf{1 3}^{\prime}$}

To a stirred solution of chiral alkynol $(10 \mathrm{mmol})$ in methanol $(20 \mathrm{~mL})$, potassium carbonate (2764 mg, $20 \mathrm{mmol})$ was added slowly at $0{ }^{\circ} \mathrm{C}$. After stirring for $20 \mathrm{~h}$ at $25^{\circ} \mathrm{C}$, water $(20 \mathrm{~mL})$ was added slowly at $0^{\circ} \mathrm{C}$. The reaction mixture was concentrated under reduced pressure. The aqueous phase was extracted with ether. The combined organic phases were washed with saturated brine solution, dried over anhydrous $\mathrm{Na}_{2} \mathrm{SO}_{4}$, concentrated under reduced pressure. The residue was purified by silica gel chromatography to get the product.

\subsubsection{Synthesis of (R)-Oct-1-yn-3-ol: (R)-12a}

Following the general procedure, the residue was purified by silica gel chromatography (hexanes/ethyl acetate $15: 1)$ to offer $(R)-\mathbf{1 2 a}\left(1098 \mathrm{mg}, 87 \%\right.$ yield) as colorless oil. $[\alpha]_{\mathrm{D}}^{25}=+16.2$ (c 1.0, ethyl ether), lit. [53] $[\alpha]_{\mathrm{D}}^{25}=+19.3$ (c 1.0, ethyl ether). HRMS ESI $[\mathrm{M}+\mathrm{Na}]^{+}$calcd for $\mathrm{C}_{8} \mathrm{H}_{14} \mathrm{NaO}^{+}$ 149.0937, found 149.0937.

\subsubsection{Synthesis of $(R)-N o n-1-y n-3-o l: ~(R)-\mathbf{1 2 b}$}

Following the general procedure, the residue was purified by silica gel chromatography (hexanes / ethyl acetate 15:1) to offer $(R)-\mathbf{1 2 b}\left(1248 \mathrm{mg}, 89 \%\right.$ yield) as colorless oil. $[\alpha]_{\mathrm{D}}^{25}=+4.1(c 2.0$, $\left.\mathrm{CHCl}_{3}\right)$, lit. [54] $[\alpha]_{\mathrm{D}}^{25}=+4.6\left(c\right.$ 1.92, $\left.\mathrm{CHCl}_{3}\right)$. HRMS ESI $[\mathrm{M}+\mathrm{Na}]^{+}$calcd for $\mathrm{C}_{9} \mathrm{H}_{16} \mathrm{NaO}^{+}$Exact mass: 163.1093, found 163.1093.

\subsubsection{Synthesis of $(R)-$ Dec-1-yn-3-ol: $(R)-12 c$}

Following the general procedure, the residue was purified by silica gel chromatography (hexanes/ethyl acetate $15: 1)$ to offer $(R)-12 \mathrm{c}(1373 \mathrm{mg}, 89 \%$ yield $)$ as colorless oil. $[\alpha]_{\mathrm{D}}^{25}=+5.1(c 1.1$, $\left.\mathrm{CHCl}_{3}\right)$, lit. [55] $[\alpha]_{\mathrm{D}}^{25}=+4.2\left(c \mathrm{1.1}, \mathrm{CHCl}_{3}\right)$. HRMS ESI $[\mathrm{M}+\mathrm{Na}]^{+}$calcd. for $\mathrm{C}_{10} \mathrm{H}_{18} \mathrm{NaO}^{+} 177.1250$, found 177.1250 .

\subsection{General Procedure for the Selective Reduction of the Chiral Alkynols $\mathbf{1 2}$}

To a stirred solution of nickel acetate tetrahydrate $(1408 \mathrm{mg}, 8 \mathrm{mmol})$ in ethanol (20 $\mathrm{mL})$ under hydrogen, sodium borohydride $(303 \mathrm{mg}, 8 \mathrm{mmol})$ in ethanol $(8 \mathrm{~mL})$ was added at $0{ }^{\circ} \mathrm{C}$. After stirring for $1 \mathrm{~h}$ at $25^{\circ} \mathrm{C}$, ethylenediamine $(481 \mathrm{mg}, 8 \mathrm{mmol})$ was added. The reaction mixture was stirred for another $10 \mathrm{~min}$ before chiral alkynol $(8 \mathrm{mmol})$ in ethanol $(8 \mathrm{~mL})$ was added slowly to the reaction mixture at $0{ }^{\circ} \mathrm{C}$. The reaction was allowed to proceed at $25^{\circ} \mathrm{C}$ under hydrogen for $6 \mathrm{~h}$ at $25^{\circ} \mathrm{C}$. The mixture was filtered through a celite pad, diluted with ether, and concentrated under reduced pressure. The residue was purified by silica gel chromatography to get the product.

\subsubsection{Synthesis of $(R)-O c t-1-e n-3-o l:(R)-6 a$}

Following the general procedure, the residue was purified by silica gel chromatography (hexanes/ethyl acetate 10:1) to offer $(R)-6 a(831 \mathrm{mg}, 81 \% \text { yield) as colorless oil. [ } \alpha]_{\mathrm{D}}^{25}=-7.3(c 1.1$, $\mathrm{CHCl}_{3}$ ), lit. [56] $[\alpha]_{\mathrm{D}}^{25}=-10.0$ (c 1.67, $\mathrm{CHCl}_{3}$ ). HRMS ESI $[\mathrm{M}+\mathrm{Na}]^{+}$calcd. for $\mathrm{C}_{8} \mathrm{H}_{16} \mathrm{NaO}^{+} 151.1093$, found 151.1093.

\subsubsection{Synthesis of (R)-Non-1-en-3-ol: $(R)-6 \mathbf{b}$}

Following the general procedure, the residue was purified by silica gel chromatography (hexanes / ethyl acetate 10:1) to offer $(R)-6 \mathbf{b}\left(944 \mathrm{mg}, 83 \%\right.$ yield) as colorless oil. $[\alpha]_{\mathrm{D}}^{25}=-10.5(c 1.1$, ethanol), lit. [57] $[\alpha]_{\mathrm{D}}^{25}=-13.4$ (c 1.12, ethanol). HRMS ESI $[\mathrm{M}+\mathrm{Na}]^{+}$calcd. for $\mathrm{C}_{9} \mathrm{H}_{18} \mathrm{NaO}^{+} 165.1250$, found 165.1250 . 


\subsubsection{Synthesis of $(R)$-Dec-1-en-3-ol: $(R)-6 \mathrm{c}$}

Following the general procedure, the residue was purified by silica gel chromatography (hexanes/ethyl acetate 10:1) to offer (R)-6c (1013 mg, 81\% yield) as colorless oil. [ $\alpha]_{\mathrm{D}}^{25}=-14.2$ (c 1.1, $\left.\mathrm{CHCl}_{3}\right)$, lit. [58] $[\alpha]_{\mathrm{D}}^{25}=-18.1\left(\right.$ c 1.22, $\left.\mathrm{CHCl}_{3}\right)$. HRMS ESI $[\mathrm{M}+\mathrm{Na}]^{+}$calcd. for $\mathrm{C}_{10} \mathrm{H}_{20} \mathrm{NaO}^{+}$ 179.1406, found 179.1406 .

\subsection{General Procedure for the Esterification Reaction and Determination of Enantiomeric Excess by HPLC}

Triethylamine (909 mg, $9 \mathrm{mmol})$ and 3,5-dinitrobenzoyl chloride (1660 mg, $7.2 \mathrm{mmol})$ were added to a stirred solution of the chiral alcohol $(6 \mathrm{mmol})$ in $\mathrm{CH}_{2} \mathrm{Cl}_{2}(40 \mathrm{~mL})$ at $-5{ }^{\circ} \mathrm{C}$. The mixture was stirred for $5 \mathrm{~h}$ at $25^{\circ} \mathrm{C}$ before water $(10 \mathrm{~mL})$ was poured into the mixture at $0{ }^{\circ} \mathrm{C}$. The aqueous phase was extracted with ether and combined organic phases were washed with saturated brine solution, dried over anhydrous $\mathrm{Na}_{2} \mathrm{SO}_{4}$, and concentrated under reduced pressure. The residue was purified by silica gel chromatography to get the product.

\subsubsection{Synthesis of (R)-Oct-1-en-3-yl-3,5-dinitrobenzoate: $(R)$-21a}

Following the general procedure, the residue was purified by silica gel chromatography (hexanes/ethyl acetate 8:1) to offer $(R)$-21a $(1837 \mathrm{mg}, 95 \%$ yield, $80 \%$ ee) as white solid. $[\alpha]_{\mathrm{D}}^{25}=-14.2\left(c 1.1, \mathrm{CH}_{2} \mathrm{Cl}_{2}\right)$. Enantiomeric excess was determined by HPLC with a Chiralcel OD-H column (99:1 $n$-hexanes:isopropanol, $1.0 \mathrm{~mL} / \mathrm{min}, 210 \mathrm{~nm}$ ), major $(R)$-enantiomer $\mathrm{t}_{\mathrm{r}}=17.80 \mathrm{~min}$, minor $(S)$-enantiomer $t_{r}=14.65 \mathrm{~min}$.

\subsubsection{Synthesis of (R)-Non-1-en-3-yl-3,5-dinitrobenzoate: $(R)$-21b}

Following the general procedure, the residue was purified by silica gel chromatography (hexanes/ethyl acetate 8:1) to offer (R)-21b (1987 $\mathrm{mg}, 98 \%$ yield, $82 \%$ ee) as colorless oil. $[\alpha]_{\mathrm{D}}^{25}=-15.2\left(c 1.1, \mathrm{CH}_{2} \mathrm{Cl}_{2}\right)$. Enantiomeric excess was determined by HPLC with a Chiralcel OD-H column (98:2 $n$-hexanes:isopropanol, $1.0 \mathrm{~mL} / \mathrm{min}, 210 \mathrm{~nm}$ ), major $(R)$-enantiomer $\mathrm{t}_{\mathrm{r}}=15.76 \mathrm{~min}$, minor $(S)$-enantiomer $t_{r}=12.93$ min.

\subsubsection{Synthesis of $(R)$-Dec-1-en-3-yl-3,5-dinitrobenzoate: $(R)$-21c}

Following the general procedure, the residue was purified by silica gel chromatography (hexanes / ethyl acetate 8:1) to offer $(R)-21 \mathrm{c}(2018 \mathrm{mg}, 96 \%$ yield, $79 \% e e)$ as white solid. $[\alpha]_{\mathrm{D}}^{25}=-18.2$ (c 1.05, $\mathrm{CH}_{2} \mathrm{Cl}_{2}$ ). Enantiomeric excess was determined by HPLC with a Chiralcel OD-H column (98:2 $n$-hexanes:isopropanol, $1.0 \mathrm{~mL} / \mathrm{min}, 210 \mathrm{~nm}$ ), major $(R)$-enantiomer $\mathrm{t}_{\mathrm{r}}=16.58 \mathrm{~min}$, minor $(S)$-enantiomer $t_{\mathrm{r}}=13.66 \mathrm{~min}$.

\subsection{General Procedure for the Recrystallization to Improve Optical Purity}

Dinitrobenzoates $(5 \mathrm{mmol})$ were dissolved in diethyl ether at room temperature, then $\mathrm{n}$-hexane was added slowly to the mixture until a white precipitate occurred. A small portion of diethyl ether was added and the white precipitate was dissolved. The mixture was cooled to $-30{ }^{\circ} \mathrm{C}$ and stayed for $48 \mathrm{~h}$ to get a white crystal of the dinitrobenzoate.

\subsubsection{Recrystallization of $(R)-O c t-1-e n-3-y l-3,5-$ dinitrobenzoate: $(R)$-21a}

Following the general procedure, the residue was purified by silica gel chromatography (hexanes/ethyl acetate 8:1) to offer (R)-21a (999 $\mathrm{mg}, 62 \%$ yield, 99\% ee) as white solid. $[\alpha]_{\mathrm{D}}^{25}=-16.1\left(c\right.$ 1.1, $\left.\mathrm{CH}_{2} \mathrm{Cl}_{2}\right)$. HRMS ESI $[\mathrm{M}+\mathrm{Na}]^{+}$calcd for $\mathrm{C}_{15} \mathrm{H}_{18} \mathrm{~N}_{2} \mathrm{NaO}_{6}{ }^{+}$345.1057, found 345.1057. Enantiomeric excess was determined by HPLC with a Chiralcel OD-H column (99:1 $n$-hexanes:isopropanol, $1.0 \mathrm{~mL} / \mathrm{min}, 210 \mathrm{~nm}$ ), major $(R)$-enantiomer $\mathrm{t}_{\mathrm{r}}=18.20 \mathrm{~min}$, minor $(S)$-enantiomer $t_{r}=14.46 \mathrm{~min}$. 


\subsubsection{Recrystallization of (R)-Non-1-en-3-yl-3,5-dinitrobenzoate: $(R)-\mathbf{2 1 b}$}

Following the general procedure, the residue was purified by silica gel chromatography (hexanes/ethyl acetate 8:1) to offer (R)-21b (1076 mg, 64\% yield, 99\% ee) as colorless oil. $[\alpha]_{\mathrm{D}}^{25}=-18.7\left(c\right.$ 1.1, $\left.\mathrm{CH}_{2} \mathrm{Cl}_{2}\right)$. HRMS ESI $[\mathrm{M}+\mathrm{Na}]^{+}$calcd for $\mathrm{C}_{16} \mathrm{H}_{20} \mathrm{~N}_{2} \mathrm{NaO}_{6}{ }^{+}$359.1214, found 359.1214. Enantiomeric excess was determined by HPLC with a Chiralcel OD-H column (98:2 $n$-hexanes:isopropanol, $1.0 \mathrm{~mL} / \mathrm{min}, 210 \mathrm{~nm})$, major $(R)$-enantiomer $\mathrm{t}_{\mathrm{r}}=15.60 \mathrm{~min}$, minor $(S)$-enantiomer $t_{\mathrm{r}}=12.67 \mathrm{~min}$.

\subsubsection{Recrystallization of $(R)$-Dec-1-en-3-yl-3,5-dinitrobenzoate: $(R)-21 \mathrm{c}$}

Following the general procedure, the residue was purified by silica gel chromatography (hexanes/ethyl acetate 8:1) to offer (R)-6c (1051 mg, 60\% yield, 99\% ee) as white solid. $[\alpha]]_{\mathrm{D}}^{25}=-21.5$ (c 1.05, $\mathrm{CH}_{2} \mathrm{Cl}_{2}$ ). HRMS ESI $[\mathrm{M}+\mathrm{Na}]^{+}$calcd for $\mathrm{C}_{17} \mathrm{H}_{22} \mathrm{~N}_{2} \mathrm{NaO}_{6}{ }^{+}$373.1370, found 373.1370. Enantiomeric excess was determined by HPLC with a Chiralcel OD-H column (98:2 n-hexanes:isopropanol, $1.0 \mathrm{~mL} / \mathrm{min}, 210 \mathrm{~nm}$ ), major $(\mathrm{R})$-enantiomer $\mathrm{t}_{\mathrm{r}}=16.46 \mathrm{~min}$, minor (S)-enantiomer $t_{r}=13.50 \mathrm{~min}$.

\section{Conclusions}

In summary, we developed a general synthetic route toward chiral matsutakeol and its analogs via the asymmetric catalytic alkynylation. A practical and efficient access was provided to prepare the (R)-matsutakeol (99\% ee, total yield up to $50.2 \%$, in three steps) and its highly enantioselective analogs, by utilizing the $(S, S)$-ProPhenol as chiral catalyst. In addition, the method may allow for gram-scale synthesis of (R)-matsutakeol and its analogs by using (S)-BINOL as chiral catalyst, thus facilitating their potential applications. Besides, this strategy has been proven to be practical for obtaining flavored allyl alcohols with high enantioselectivity in food analysis and screening insect attractants. Biological evaluation of target molecules is in progress and will be reported in due course.

Supplementary Materials: The following are available online: Figure S1-S43.

Acknowledgments: We thank the Special Fund for Agroscientific Research in the Public Interest (No.201503112), National Natural Science Foundation of China (No.21602043 and 31601657), the Agricultural Technology System of Henan (No.S2014-11-G03), Science and Technology Planning Project of Henan Province of China (No.142102110050) and (No.141PPTGG424), the Educational Commission of Henan Province of China (No.14A210027), Henan Key Laboratory of the Innovation and Application of Novel Pesticide, Collaborative Innovation Center of Henan Grain Crops, National Key Laboratory of Wheat and Maize Crop Science for the financial support of this study.

Author Contributions: This research was carried on by all the authors. Chao Zheng, Shichao Lu, Jia Liu, Risong Na, and Bin Yu performed the experiments; Honglian Li, Xianru Guo, Xinming Yin, and Bin Yu participated in bio-activity experiment; Jia Liu and Min Wang wrote the paper.

Conflicts of Interest: The authors declare no conflict of interest.

\section{References}

1. Negri, R. Polyacetylenes from terrestrial plants and fungi: Recent phytochemical and biological advances. Fitoterapia 2015, 106, 92-109. [CrossRef] [PubMed]

2. Konovalov, D.A. Polyacetylene compounds of plants of the Asteraceae Family (Review). Pharm. Chem. J. 2014, 48, 613-631. [CrossRef]

3. Finimundy, T.C.; Dillon, A.J.P.; Henriques, J.A.P.; Ely, M.R. A review on general nutritional compounds and pharmacological properties of the Lentinula edodes mushroom. Food Nutr. Sci. 2014, 5, 1095-1105. [CrossRef]

4. Swift, K.A.D. Current Topics in Flavors and Fragrances: Towards a New Millennium of Discovery; Springer Science + Business Media B.V.: GX Dordrecht, The Netherlands, 1999; p. 235.

5. Mizuno, T. Bioactive biomolecules of mushrooms: Food function and medicinal effect of mushroom fungi. Food Rev. Int. 1995, 11, 7-21. [CrossRef] 
6. Murahashi, S. The odor of matsutake (Armillaria matsutake Ito et Imai Agaricaceae). II. Sci. Papers Inst. Phys. Chem. Res. 1938, 34, 155-172.

7. Hung, R.; Lee, S.; Bennett, J.W. The effects of low concentrations of the enantiomers of mushroom alcohol (1-octen-3-ol) on Arabidopsis thaliana. Mycology 2014, 5, 73-80. [CrossRef] [PubMed]

8. McMeniman, C.J.; Corfas, R.A.; Matthews, B.J.; Ritchie, S.A.; Vosshall, L.B. Multimodal Integration of Carbon Dioxide and Other Sensory Cues Drives Mosquito Attraction to Humans. Cell 2014, 156, 1060-1071. [CrossRef] [PubMed]

9. Sikuljak, T.; Mazuir, F.; Arevalo, A.; Menon, A. Pesticidal Mixture Comprising a Carboxamide Compound and a Biopesticide. WO2016142456 A1, 10 March 2016.

10. Combet, E.; Henderson, J.; Eastwood, D.C.; Burton, K.S. Eight-carbon volatiles in mushrooms and fungi: Properties, analysis, and biosynthesis. Mycoscience 2006, 47, 317-326. [CrossRef]

11. Helmchen, G.; Ihrig, K.; Schindler, H. EPC-syntheses via asymmetric Diels-Alder reactions/retro Diels-Alder reactions. I. $(R)$ - and $(S)$-Matsutake alcohol, $(R)$ - and $(S)$-sarcomycin methyl ester. Tetrahedron Lett. 1987, 28, 183-186. [CrossRef]

12. Takano, S.; Yanase, M.; Takahashi, M.; Ogasawara, K. Enantiodivergent synthesis of both enantiomers of sulcatol and matsutake alcohol from (R)-epichlorohydrin. Chem. Lett. 1987, 16, 2017-2020. [CrossRef]

13. Kitamura, M.; Kasahara, I.; Manabe, K.; Noyori, R.; Takaya, H. Kinetic resolution of racemic allylic alcohols by BINAP-ruthenium(II) catalyzed hydrogenation. J. Org. Chem. 1988, 53, 708-710. [CrossRef]

14. Oppolzer, W.; Radinov, R.N. Enantioselective synthesis of sec-allyl alcohols by catalytic asymmetric addition of divinylzinc to aldehydes. Tetrahedron Lett. 1988, 29, 5645-5648. [CrossRef]

15. Kusuda, S.; Ueno, Y.; Toru, T. Vinyl anion equivalent V. Asymmetric synthesis of allylic alcohols using chiral 2-(trialkylsilyl)ethyl sulfoxides. Tetrahedron 1994, 50, 1045-1062. [CrossRef]

16. Bisogno, F.R.; Rioz-Martinez, A.; Rodriguez, C.; Lavandera, I.; de Gonzalo, G.; Torres Pazmino, D.E.; Fraaije, M.W.; Gotor, V. Oxidoreductases Working Together: Concurrent Obtaining of Valuable Derivatives by Employing the PIKAT Method. ChemCatChem 2010, 2, 946-949. [CrossRef]

17. Rej, R.K.; Nanda, S. Chemoenzymic Asymmetric Total Synthesis of Nonanolide (Z)-Cytospolides D, E and Their Stereoisomers. Eur. J. Org. Chem. 2014, 2014, 860-871. [CrossRef]

18. Rej, R.K.; Kumar, R.; Nanda, S. Asymmetric synthesis of cytospolide C and cytospolide D through successful exploration of stereoselective Julia-Kocienski olefination and Suzuki reaction followed by macrolactonization. Tetrahedron 2015, 71, 3185-3194. [CrossRef]

19. Lee, W.H.; Bae, I.H.; Kim, B.M.; Seu, Y.-B. New Synthetic Approach to Optically Activate Matsutakeol, the Major Flavor Component of Tricholoma matsutake, from L-Tartaric Acid. Bull. Korean Chem. Soc. 2016, 37, 1910-1911. [CrossRef]

20. Huang, J.; Wei, S.; Wang, L.; Zhang, C.; Li, S.; Liu, P.; Du, X.; Wang, Q. Highly enantioselective catalytic methyl propiolate addition to both aromatic and aliphatic aldehydes. Tetrahedron Asymmetry 2016, 27, 428-435. [CrossRef]

21. Funes-Maldonado, M.; Sieng, B.; Amedjkouh, M. Enabling Asymmetric Alkynylation of Azaaryl Aldehydes with Soai Autocatalyst. Eur. J. Org. Chem. 2015, 2015, 4081-4086. [CrossRef]

22. Yang, Y.-Q.; Li, S.-N.; Zhong, J.-C.; Zhou, Y.; Zeng, H.-Z.; Duan, H.-J.; Bian, Q.-H.; Wang, M. Total synthesis of each enantiomer of falcarinol and panaxjapyne A via asymmetric catalytic alkynylation of an aldehyde. Tetrahedron Asymmetry 2015, 26, 361-366. [CrossRef]

23. Trost, B.M.; Quintard, A. Asymmetric Catalytic Alkynylation of Acetaldehyde: Application to the Synthesis of (+)-Tetrahydropyrenophorol. Angew. Chem. Int. Ed. 2012, 51, 6704-6708. [CrossRef] [PubMed]

24. Trost, B.M.; Burns, A.C.; Bartlett, M.J.; Tautz, T.; Weiss, A.H. Thionium Ion Initiated Medium-Sized Ring Formation: The Total Synthesis of Asteriscunolide D. J. Am. Chem. Soc. 2012, 134, 1474-1477. [CrossRef] [PubMed]

25. Trost, B.M.; Bartlett, M.J.; Weiss, A.H.; von Wangelin, A.J.; Chan, V.S. Development of Zn-ProPhenol-Catalyzed Asymmetric Alkyne Addition: Synthesis of Chiral Propargylic Alcohols. Chem. Eur. J. 2012, 18, 16498-16509. [CrossRef] [PubMed]

26. Trost, B.M.; Bartlett, M.J. Transition-Metal-Catalyzed Synthesis of Aspergillide B: An Alkyne Addition Strategy. Org. Lett. 2012, 14, 1322-1325. [CrossRef] [PubMed] 
27. Na, R.; Wang, B.; Liu, H.; Bian, Q.; Zhong, J.; Wang, M.; Guo, H. Synthesis and application of novel dithiane alcohol ligand based on chiral cyclopropane-backbone for asymmetric ethylation of aromatic aldehydes. Lett. Org. Chem. 2012, 9, 622-627. [CrossRef]

28. Chen, X.; Chen, W.; Wang, L.; Yu, X.-Q.; Huang, D.-S.; Pu, L. Synthesis of a C1 symmetric BINOL-terpyridine ligand and highly enantioselective methyl propiolate addition to aromatic aldehydes. Tetrahedron 2010, 66, 1990-1993. [CrossRef]

29. Ito, J.-I.; Asai, R.; Nishiyama, H. Asymmetric Direct Alkynylation Catalyzed by Chiral Ru-Bis(oxazolinyl)phenyl Complexes. Org. Lett. 2010, 12, 3860-3862. [CrossRef] [PubMed]

30. Turlington, M.; DeBerardinis, A.M.; Pu, L. Highly Enantioselective Catalytic Alkyl Propiolate Addition to Aliphatic Aldehydes. Org. Lett. 2009, 11, 2441-2444. [CrossRef] [PubMed]

31. Lin, L.; Zhao, Q.; Li, A.N.; Ren, F.; Yang, F.; Wang, R. Enantioselective synthesis of Anomala osakana pheromone and Janus integer pheromone: A flexible approach to chiral $\boldsymbol{\gamma}$-butyrolactones. Org. Biomol. Chem. 2009, 7, 3663-3665. [CrossRef] [PubMed]

32. Qiu, L.; Wang, Q.; Lin, L.; Liu, X.; Jiang, X.; Zhao, Q.; Hu, G.; Wang, R. Highly enantioselective addition of terminal alkynes to aldehydes catalyzed by a new chiral $\beta$-sulfonamide alcohol/Ti(OiPr) $4 / \mathrm{Et} 2 \mathrm{Zn} / \mathrm{R} 3 \mathrm{~N}$ catalyst system. Chirality 2009, 21, 316-323. [CrossRef] [PubMed]

33. Zhong, J.-C.; Hou, S.-C.; Bian, Q.-H.; Yin, M.-M.; Na, R.-S.; Zheng, B.; Li, Z.-Y.; Liu, S.-Z.; Wang, M. Highly enantioselective zinc/amino alcohol-catalyzed alkynylation of aldehydes. Chem. Eur. J. 2009, 15, 3069-3071. [CrossRef] [PubMed]

34. Yue, Y.; Turlington, M.; Yu, X.-Q.; Pu, L. 3,3'-Anisyl-Substituted BINOL, H4BINOL, and H8BINOL Ligands: Asymmetric Synthesis of Diverse Propargylic Alcohols and Their Ring-Closing Metathesis to Chiral Cycloalkenes. J. Org. Chem. 2009, 74, 8681-8689. [CrossRef] [PubMed]

35. Xu, Z.; Wu, N.; Ding, Z.; Wang, T.; Mao, J.; Zhang, Y. L-Proline-derived tertiary amino alcohol as a new chiral ligand for enantioselective alkynylation of aldehydes. Tetrahedron Lett. 2009, 50, 926-929. [CrossRef]

36. Yang, F.; Xi, P.; Yang, L.; Lan, J.; Xie, R.; You, J. Facile, Mild, and Highly Enantioselective Alkynylzinc Addition to Aromatic Aldehydes by BINOL/N-Methylimidazole Dual Catalysis. J. Org. Chem. 2007, 72, 5457-5460. [CrossRef] [PubMed]

37. Lin, L.; Jiang, X.; Liu, W.; Qiu, L.; Xu, Z.; Xu, J.; Chan, A.S.C.; Wang, R. Highly Enantioselective Synthesis of $\gamma$-Hydroxy- $\alpha, \beta$-acetylenic Esters Catalyzed by a $\beta$-Sulfonamide Alcohol. Org. Lett. 2007, 9, 2329-2332. [CrossRef] [PubMed]

38. Trost, B.M.; Weiss, A.H. Catalytic Enantioselective Synthesis of Adociacetylene B. Org. Lett. 2006, 8, 4461-4464. [CrossRef] [PubMed]

39. Trost, B.M.; Weiss, A.H.; von Wangelin, A.J. Dinuclear Zn-Catalyzed Asymmetric Alkynylation of Unsaturated Aldehydes. J. Am. Chem. Soc. 2006, 128, 8-9. [CrossRef] [PubMed]

40. Gao, G.; Wang, Q.; Yu, X.-Q.; Xie, R.-G.; Pu, L. Highly enantioselective synthesis of $\gamma$-hydroxy- $\alpha, \beta$-acetylenic esters by asymmetric alkyne addition to aldehydes. Angew. Chem. Int. Ed. 2006, 45, 122-125. [CrossRef] [PubMed]

41. Wolf, C.; Liu, S. Bisoxazolidine-catalyzed enantioselective alkynylation of aldehydes. J. Am. Chem. Soc. 2006, 128, 10996-10997. [CrossRef] [PubMed]

42. Emmerson, D.P.G.; Hems, W.P.; Davis, B.G. Carbohydrate-Derived Amino-Alcohol Ligands for Asymmetric Alkynylation of Aldehydes. Org. Lett. 2006, 8, 207-210. [CrossRef] [PubMed]

43. Cozzi, P.G.; Rudolph, J.; Bolm, C.; Norrby, P.-O.; Tomasini, C. Me 2 Zn-Mediated Addition of Acetylenes to Aldehydes and Ketones. J. Org. Chem. 2005, 70, 5733-5736. [CrossRef] [PubMed]

44. Yamashita, M.; Yamada, K.-i.; Tomioka, K. Catalytic asymmetric addition of terminal alkynes to aldehydes mediated by (1R,2R)-2-(dimethylamino)-1,2-diphenylethanol. Adv. Synth. Catal. 2005, 347, 1649-1652. [CrossRef]

45. Jiang, B.; Si, Y.-G. The first highly enantioselective alkynylation of chloral: A practical and efficient pathway to chiral trichloromethyl propargyl alcohols. Adv. Synth. Catal. 2004, 346, 669-674. [CrossRef]

46. Dahmen, S. Enantioselective Alkynylation of Aldehydes Catalyzed by [2.2]Paracyclophane-Based Ligands. Org. Lett. 2004, 6, 2113-2116. [CrossRef] [PubMed]

47. Stefani, H.A.; Menezes, P.H.; Costa, I.M.; Silva, D.O.; Petragnani, N. Use of chiral sulfoxide in the asymmetric synthesis of (+)-virol C. Synlett 2002, 8, 1335-1337. [CrossRef] 
48. Jiang, B.; Chen, Z.; Xiong, W. Highly enantioselective alkynylation of aldehydes catalyzed by a readily available chiral amino alcohol-based ligand. Chem. Commun. (Camb., UK) 2002, 14, 1524-1525. [CrossRef]

49. Rotticci, D.; Haeffner, F.; Orrenius, C.; Norin, T.; Hult, K. Molecular recognition of sec-alcohol enantiomers by Candida antarctica lipase B. J. Mol. Catal. B Enzym. 1998, 5, 267-272. [CrossRef]

50. Liu, J.; Li, H.-L.; Guo, X.-R.; Zhou, L.; Wang, Y.; Duan, Y.-N.; Wang, M.-Z.; Na, R.-S.; Yu, B. A general strategy toward the total synthesis of C17 polyacetylenes virols A and C. Tetrahedron 2016, 72, 6603-6610. [CrossRef]

51. Morandi, S.; Palate, F.; Benvenuti, S.; Prati, F. Total synthesis of a dienynone from Echinacea pallida. Tetrahedron 2008, 64, 6324-6328. [CrossRef]

52. Zheng, M.; Wu, F.; Chen, K.; Zhu, S. Styrene as $4 \pi$-Component in Zn (II)-Catalyzed Intermolecular Diels-Alder/Ene Tandem Reaction. Org. Lett. 2016, 18, 3554-3557. [CrossRef] [PubMed]

53. Johnson, W.S.; Elliott, R.; Elliott, J.D. Asymmetric synthesis via acetal templates. 4. Reactions with silylacetylenic compounds. Formation of chiral propargylic alcohols. J. Am. Chem. Soc. 1983, 105, 2904-2905. [CrossRef]

54. Jiang, X.; Fu, C.; Ma, S. Highly stereoselective iodolactonization of 4,5-allenoic acids-An efficient synthesis of 5-(1'-iodo-1'(Z)-alkenyl)-4,5-dihydro-2(3H)-furanones. Chemistry 2008, 14, 9656-9664. [CrossRef] [PubMed]

55. Fu, R.; Chen, J.; Guo, L.-C.; Ye, J.-L.; Ruan, Y.-P.; Huang, P.-Q. Asymmetric Total Synthesis of (-)-Awajanomycin. Org. Lett. 2009, 11, 5242-5245. [CrossRef] [PubMed]

56. Nakamura, S.; Nakayama, J.; Toru, T. Asymmetric Reduction of $\boldsymbol{\alpha}$-(Trimethylsilyl)methyl- $\beta$-ketosulfoxide with DIBAL under Basic Conditions. J. Org. Chem. 2003, 68, 5766-5768. [CrossRef] [PubMed]

57. Bessodes, M.; Abushanab, E.; Antonakis, K. Enantiospecific synthesis of the immunopotentiators erythro-9-(2-hydroxy-3-nonyl)hypoxanthines and the threo-diastereomers. Tetrahedron Lett. 1984, 25, 5899-5902. [CrossRef]

58. Gartner, M.; Mader, S.; Seehafer, K.; Helmchen, G. Enantio- and Regioselective Iridium-Catalyzed Allylic Hydroxylation. J. Am. Chem. Soc. 2011, 133, 2072-2075. [CrossRef] [PubMed]

Sample Availability: Samples of (R)-Matsutakeol and compounds 13a-i are available from the authors.

(C) 2017 by the authors. Licensee MDPI, Basel, Switzerland. This article is an open access article distributed under the terms and conditions of the Creative Commons Attribution (CC BY) license (http:/ / creativecommons.org/licenses/by/4.0/). 